\title{
Skull Variability of Martes martes and Martes foina from Poland
}

\author{
Santiago REIG ${ }^{1}$ \& Andrzej L. RUPRECHT
}

Reig S. \& Ruprecht A.L., 1989: Skull variability of $M$. martes and $M$. foina from Poland. Acta theriol., 34, 41: 595-624 [With 13 Tables \& 11 Figs]

A morphological study of sex, age, and population variation of Martes martes (Linnaeus, 1758) and Martes foina (Erxleben, 1777) was completed, using a collection of 236 and 47 skulls, respectively. Twenty-three measurements were taken on each specimen. Three age groups (juveniles, subadults and aduits) were recognized, based on relative criteria such as suture obliteration, tooth wear, and development of crista sagittalis. Univariate and multivariate techniques were used to process the data. Sex dimorphism was clear, males being on average $12.1 \%$ and $9.5 \%$ larger than females in $M$. martes and $M$. foina, respectively. Masticatory apparatus was the region of the skull showing more significant sex dimorphism in both species. Statistical significance of sexual dimorphism in particular traits was distinet in both species. Weight and length of the mandible, and zygomatic width showed the highest differences among age classes in both species. In $M$. martes two principal factors explained more than $70 \%$ of the total age variation; the first one was related to length of the skull, accounting for most of the variance (more than $50 \%$ ), and the second to width of the skull. However in $M$. foina the total amount of variation explained was also high but the relative importance of the length and width factors was similar. Rostral angle was the best character to classify the skulls into species. Using a dividing point of $54.9^{\circ}$, below which specimens should be classified as $M$. martes and vice versa, $98 \%$ of the skulls were correctly classified. $100 \%$ correct classification of Polish martens was achieved by a discriminant function which used only five skull measurements. Separation of species was also possible by plotting of two principal factors defining a length and width component of skull variation in each sex. Differences between four populations (Pomerania and Masurian Lake Region; Wielkopolska-Kujawy Lowlands; Bialowieża Primeval Forest; Lower and Upper Silesia) were studied in $M$. mar tes. Significant differences were found in eleven measurements, skulls from Białowieża being smaller than from the other three populations. Most of the morphological changes in the skull of martens due to sex, age or species variation were explained by the association of measurements into a length and a width factor. However, in each species the relative importance of these factors to explain the variability observed was different. Phylogenetic and ecological reasons are the only possible explanation for different patterns of variation in the two marten species.

[Mammal Research Institute, Polish Academy of Sciences, 17-230 Bialowieża, Poland]

\section{INTRODUCTION}

Earlier studies on the morphological characteristics of the pine marten Martes martes (Linnaeus, 1758) and the stone marten $M$. foina (Erxleben, 1777) have been dealing with the taxonomic characters

1 Present address: Museo Nacional de Ciencias Naturales, CSIC, J. Gutierrez Abascal 2, 28006 Madrid, Spain 
which allow distinction between the two species (see Anderson, 1970 for review). Attempts to find diagnostic traits are still being made using skull measurements (Altuna, 1973), non metrical characters (Steiner \& Steiner, 1986) or multivariate methods (Gerasimov, 1985). Other morphological studies on European martens deal with criteria for age determination (Röttcher, 1965; Habermehl \& Röttcher, 1967), sex dimorphism (Bree et al., 1970; Rossolimo \& Pavlinov, 1974), or variations in dental morphology (Wolsan et al., 1985, 1986). However, there is no complete information on the morphological pattern of variation of these species related to sex, age, or population differences.

Comparative study of morphological variation in $M$. martes and $M$. foina is particularly interesting because they have strong morphological similarities and at the same time exhibit noticeable differences concerning their ecology and especially the habitat preferences. $M$. martes is more selective for the choice of habitat, being associated with mature coniferous forest (Heptner et al., 1967; Pulliainen, 1981a), while $M$. foina inhabits either mixed or coniferous forest, rocky hillsides, and often being close to human dwellings (Heptner et al., 1967). The aim of this paper is to analyze the variation of the skull related to sex and age changes, the differences between populations and the differences between both species.

\section{MATERLAL AND METHODS}

This study is based on 236 skulls of $M$. martes and 47 of $M$. foina. Specimens were obtained from the Mammals Research Institute PAS (Białowieza), Institute of Systematic and Experimental Zoology PAS (Cracow), and the private collection of A. L. Ruprecht.

\subsection{Age Classes}

To avoid interferences due to age variation and to analyze the growth changes in the skull without splitting the material too much, three relative age classes were considered: Juveniles, Subadults and Adults. The criteria followed for this grouping were similar to those used by Buchalczyk \& Ruprecht (1977) for Mustela putorius Linnaeus, 1758. These include the date of killing, obliteration of sutura internasalis and nasomaxillaris, general aspect of the skull (development of crista sagittalis, appearance of skull bone), degree of "denudation" of the canine teeth, and tooth wear.

Age class 1 (Juveniles) included martens killed from September to January, already with permanent teeth, clearly marked sutura intemasalis and nasomaxillaris, and rugged aspect of the skull bones. Assuming that birth period takes place in May (Ryabov, 1962), the approximate age of this group was from five to eight months.

Age class II (Subadults) includes animals killed between February and April, with slightly visible sutures, and noticeable development of crista sagittalis. The approximate age was from nine to twelve months.

Age class III (AduIts) corresponds to specimens killed after March of their first year, having smooth bone surface, sutures completely obliterated, well developed crista sagittalis and visible tooth wear. These martens were considered to be older than one year. 


\subsection{Cranial Measurements}

Twenty three measurements commonly used in mustelids (Anderson, 1970; Buchalczyk \& Ruprecht, 1977) were performed on each specimen, using vernier caliper with an accuracy of $0.1 \mathrm{~mm}$. These were the following:

Length measurements ( $\mathrm{mm}$ ):

1. Condylobasal length

2. Profile length

3. Facial length

4. Braincase length

5. Condylo-molar length

6. Maxillary tooth-row length

7. Palatal length

Width measurements $(\mathrm{mm})$ :

8. Zygomatic width

9. Ectoorbital width

10. Interorbital constriction

11. Postorbital width

12. Mastoid width

13. Bimolar width

14. Rostrum width
Mandible measurements (mm):

$\begin{array}{lll}\text { (CbL) } & \text { 15. Lower canine length } & \text { (LcL) } \\ \text { (PrL) } & \text { 16. First lower molar length } & \text { (LmL) } \\ \text { (FcL) } & \text { 17. Mandibular tooth-row length } & (\mathrm{MntL}) \\ \text { (BcL) } & \text { 18. Mandible height } & \text { (MnbH) } \\ \text { (CmL) } & \text { 19. Mandible length } & \text { (MnbL) } \\ \text { (MxtL) } & \text { 20. Mandible weight (g) } & \text { (MnbW) }\end{array}$

(MxtL)

20. Mandible weight (g)

\section{Others:}

\section{(ZyW)}

(EorW)

(IorW)

(PorW)

(MstW)

(BmW)

(RtrW)

21. Braincase height ( $\mathrm{mm}$ )

22. Braincase capacity (cc)

(BcbH)

23. Rostral angle (')

(BcC)

(RstA)

Definitions of particular measurements are clearly ascertained from enclosed figures (Fig. 1). RstA was measured following the procedure of Ruprecht (1972). BcC was measured by filling the braincase with fine shot $(1.5 \mathrm{~mm} \phi)$. To increase accuracy, the shot needed to fill the braincase cavity was weighed instead of measuring its volume (1cc $=6.3$ g).

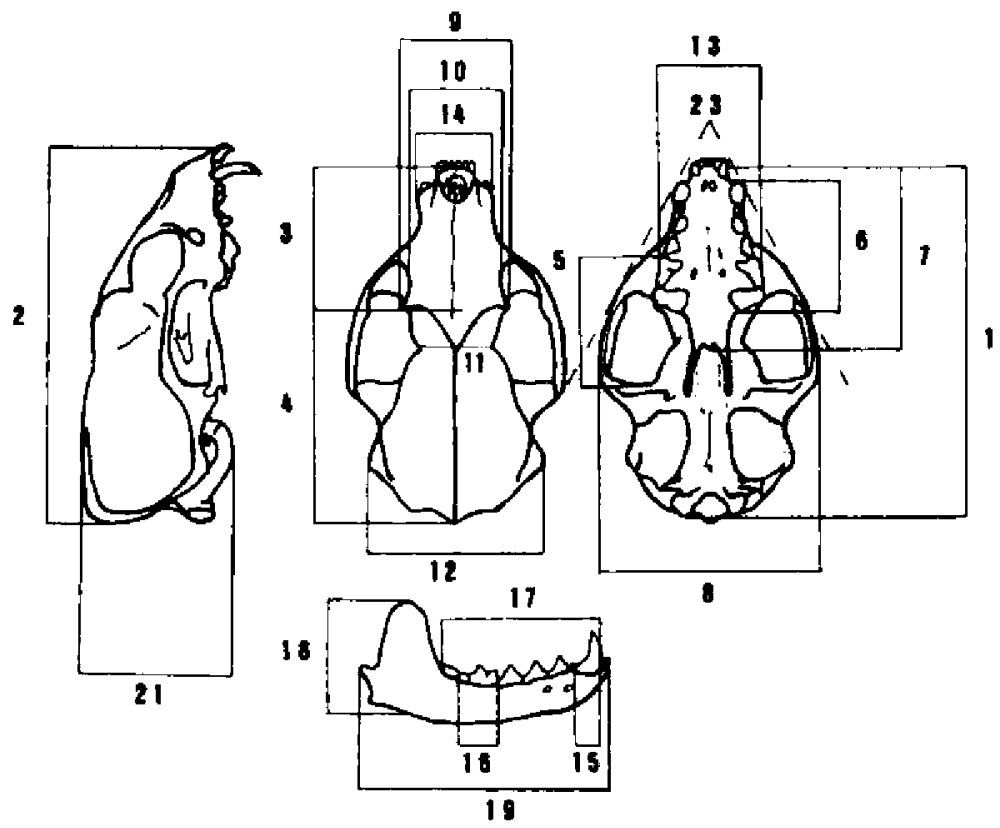

Fig. 1. Skull measuraments used in this study. Numbers indicate measurements listed in the text. 
In addition to single measurements, ratios between two of them were calculated. Only those which showed greater significance than single characters in the differences among groups were mentioned in the results, as indicators of changes in skull proportions.

\subsection{Population Groups}

Differences among populations within the Polish territory were analyzed only in $M$. martes because of the larger number of skulls available. The material examined, adult males only, was grouped into four regions of particular faunistic and geographic significance: (1) Pomerania and Masurian Lake Region; (2) Wielkopolska-Kujawy Lowlands; (3) Białowieża Primeval Forest; (4) Lower and Upper Silesia.

\subsection{Statistical Analysis}

Sample statistics were calculated separately for each group considered (age class, sex, species). The simplest parameter used for comparison among groups was the percentage difference between means (\%D), calculated relative to the group showing the smallest value $\left[\% \mathrm{D}=(\right.$ largest value-smallest value/smalest $\times 100)$. Mean of $\% \mathrm{D}\left(\overline{\mathrm{x}}_{\% \mathrm{D}}\right)$ among the 23 measurements was also calculated, being elucidated as the average size difference between the groups compared. All calculations were made using the computer package BMDP87 (Dixon, 1987).

Univariate methods were mainly restricted to analysis of variance (ANOVA), which tests the significance of the observed variation in individual characters among the respective groups (age class, sex, populations, species). Due to unequal sample size, Levene's test for equality of variances was performed simultaneously to asses the validity of this analysis (BMDP7D, Dixon, 1987). When the results of ANOVA are given, the $F$ value expresses the degree of significance of the differences between groups beyond the $p<0.001$ level. Compared to $\% D, F$ epresents to what extent the percentage of differences found among the groups considered (age, sex, species) is due to hazard or not. Attention must be paid to the fact that $F$ value is n-dependent, and hence, comparison of this value with other $F$ obtained from a test performed in a group of different sample size is inappropriate because the degrees of freedom are not equivalent. Other univariate techniques used include pair-wise comparison between group means by Student's $t$ test.

Multivariate analysis was carried out to examine variation from a multidimensional point of view (Sokal \& Rinkel, 1963; Gould \& Johnston, 1972). Stepwise discriminant analysis (BMDP7M, Dixon, 1987) was ued to construct from the original variables, a discriminant function (represented as canonical variables) which maximally separates the groups analyzed (sex, species). Association between measurements was investigated by Principal Factor Analysis (BMDP4M, Dixon, 1987) (Atchley, 1971b; Baker et al., 1978, Wiig, 1985). This technique transforms the original set of variables into factors that join those measurements having a similar and independent variability pattern. Therefore, factors are characterized by different (opposite) loading's from each variable. Factors were subjected to an orthogonal rotation (Varimax) in order to facilitate interpretation (Atchley, 1971b; Baker et al., 1978).

Since multivariate methods require complete sets of data, missing values of each specimen were estimated by stepwise regression from the remaining set (minimum of $60 \%$ ) of variables available for this specimen (BMDPAM, Dixon, 1987). This estimation was made separately for age class, sex, species and populations. The predicted values were then replaced in the data matrix. All variables were tested for skewness and kurtosis. When appropriate, data transformation by Log function were used to approach distribution to normality. 


\section{RESULTS}

Sample statistics for all groups of Polish skulls are depicted in Tables 1 and 2. No remarkable differences were found between species, age, or sex groups, regarding CV. Characters more variable were those measuring non linear traits, as weight of the mandible or volume of the braincase. The remaining measurements exhibited similar $\mathrm{CV}$, those related to width of the skull having little higher CV than length ones. Variability of postorbital width was very high in both species.

\subsection{Sex Dimorphism}

In both species, sex dimorphism was apparent, males being larger than females, although overlapping within the observed range of variation occurred in most measurements (Tables 1,2 ). Univariate analysis was performed in both species using the group of adults only. Results obtained from ANOVA revealed significant differences $(p<0.001)$ between sexes in both species and in all measurements except RstA (Table 3).

Measurements related to the mandible showed the most significant sex differences, having little or no overlapping (Tables 1,2), MnbL and $\mathrm{MnbH}$ reaching the highest $F$ value in $M$. martes and $M$. foina, respectively (Table 3 ). $\mathrm{CbL}, \mathrm{PrL}$, and $\mathrm{CmL}$ also had very high $F$ value indicating that sexual dimorphism was more apparent in length than in width in both species. RtrW and $\mathrm{ZyW}$ also showed high significant sex differences (Table 3).

Sex dimorphism was more strong in $M$. martes than in $M$. foina. On an average, females were 12.1 and $9.5 \%$ smaller than males in $M$. martes and $M$. foina respectively (Table 3 ). In both species, strongest dimorphism was found in those traits related to size and weight of the mandible. On the contrary, $\mathrm{BmW}$ was the character more similar in males and females (Table 3 ).

Distinctive sex differences in the development of the crista sagittalis were observed, and consequently, related measurements like $\mathrm{BcbH}$ showed also high significant sex dimorphism, specially in $M$. foina (Table 3).

Character ratios examined were found to have low significance in sex dimorphism in comparison to single measurements, even though they include those traits with high $F$ values. The indices MndW/PrL, MndW/MndL (Heptner \& Morozova-Turova, 1951) and BcbH/MndW (Buchalczyk \& Ruprecht, 1977) showed the greatest changes among sexes (Table 3). In particular, MndW and PrL, when plotted against each other clearly separate adult males and females in both species (Figs. 2, 3). 
Table 1

Sample statistics of the 23 skull measurements performed (see the text for explanation $C$. V.: coefficient of variation, OR.: observed range, $\bar{X}_{c y}$ : mean of the coefficient of given. Actual sample size of particular measurements is on average $92 \%$ of the number

\begin{tabular}{|c|c|c|c|c|c|c|c|c|c|}
\hline & \multicolumn{9}{|c|}{ Females $(n=103)$} \\
\hline & \multicolumn{3}{|c|}{ Juveniles $(n=44$} & \multicolumn{3}{|c|}{ Subadults $(n=34)$} & \multicolumn{3}{|c|}{ Adults $(n=25)$} \\
\hline & $\overline{\overline{\mathbf{x}}}$ & C. V. & O. $\mathbf{R}$ & $\overline{\overline{\mathbf{x}}}$ & c. V. & O. $\mathbf{R}$ & $\overline{\overline{\mathbf{x}}}$ & C. V. & O. R \\
\hline $\begin{array}{l}\text { CbL } \\
\text { PrL } \\
\text { FcL } \\
\text { BcL } \\
\text { CmL } \\
\text { MxtL } \\
\text { PtL }\end{array}$ & $\begin{array}{l}76.8 \\
87.6 \\
35.0 \\
49.7 \\
30.6 \\
27.2 \\
37.4\end{array}$ & $\begin{array}{l}2.2 \\
2.3 \\
3.2 \\
2.5 \\
2.6 \\
5.4 \\
2.5\end{array}$ & $\begin{array}{l}72.0-80.2 \\
73.6-81.8 \\
32.2-37.3 \\
47.6-52.9 \\
28.0-32.0 \\
22.8-32.3 \\
34.6-39.6\end{array}$ & $\begin{array}{l}78.0 \\
79.8 \\
36.0 \\
49.7 \\
31.3 \\
27.7 \\
38.0\end{array}$ & $\begin{array}{l}1.7 \\
1.8 \\
3.0 \\
2.5 \\
2.3 \\
4.1 \\
2.1\end{array}$ & $\begin{array}{l}75.3-80.6 \\
75.5-82.3 \\
33.5-37.8 \\
46.2-52.0 \\
29.4-33.1 \\
22.4-28.9 \\
36.3-39.8\end{array}$ & $\begin{array}{l}78.8 \\
81.0 \\
36.2 \\
50.9 \\
31.6 \\
28.0 \\
38.2\end{array}$ & $\begin{array}{l}1.8 \\
2.0 \\
2.7 \\
2.6 \\
2.5 \\
4.4 \\
2.4\end{array}$ & $\begin{array}{l}75.5-81.6 \\
76.8-83.5 \\
34.3-37.8 \\
47.2-53.4 \\
30.0-33.0 \\
22.7-29.1 \\
36.0-39.7\end{array}$ \\
\hline $\begin{array}{l}\text { ZyW } \\
\text { EorW } \\
\text { IorW } \\
\text { PorW } \\
\text { MstW } \\
\text { BmW } \\
\text { RtrW }\end{array}$ & $\begin{array}{l}43.4 \\
22.0 \\
18.7 \\
18.4 \\
36.1 \\
21.3 \\
15.3\end{array}$ & $\begin{array}{l}2.7 \\
3.1 \\
4.7 \\
6.3 \\
2.8 \\
3.4 \\
4.3\end{array}$ & $\begin{array}{l}40.1-45.3 \\
19.1-23.5 \\
17.0-20.3 \\
15.9-20.8 \\
33.8-38.2 \\
19.3-22.8 \\
14.4-17.9\end{array}$ & $\begin{array}{l}44.6 \\
23.1 \\
19.4 \\
18.5 \\
36.8 \\
21.7 \\
15.6\end{array}$ & $\begin{array}{l}2.7 \\
5.1 \\
4.1 \\
6.3 \\
2.9 \\
4.0 \\
3.0\end{array}$ & $\begin{array}{l}41.9-46.4 \\
20.7-25.7 \\
18.0-21.4 \\
15.8-20.3 \\
33.0-38.2 \\
20.2-23.2 \\
15.0-16.7\end{array}$ & $\begin{array}{l}45.3 \\
22.9 \\
19.4 \\
17.3 \\
37.3 \\
22.1 \\
16.0\end{array}$ & $\begin{array}{l}2.4 \\
3.4 \\
3.8 \\
6.3 \\
3.0 \\
4.7 \\
3.1\end{array}$ & $\begin{array}{l}43.0-48.0 \\
21.9-24.7 \\
18.4-21.2 \\
15.3-20.0 \\
34.4-39.3 \\
20.0-24.0 \\
14.8-17.0\end{array}$ \\
\hline $\begin{array}{l}\text { LcL } \\
\text { LmL } \\
\text { MntL } \\
\text { MnbH } \\
\text { MnbL } \\
\text { MnbW }\end{array}$ & $\begin{array}{r}4.2 \\
9.0 \\
31.4 \\
21.4 \\
50.5 \\
4.3\end{array}$ & $\begin{array}{l}4.2 \\
3.8 \\
2.3 \\
4.0 \\
2.5 \\
9.7\end{array}$ & $\begin{array}{c}3.9-4.6 \\
8.3-9.9 \\
29.6-32.9 \\
19.3-23.0 \\
46.3-52.4 \\
3.5-5.2\end{array}$ & $\begin{array}{r}4.3 \\
9.1 \\
32.1 \\
22.0 \\
52.0 \\
4.6\end{array}$ & $\begin{array}{l}4.6 \\
3.4 \\
2.2 \\
3.7 \\
2.1 \\
9.1\end{array}$ & $\begin{array}{c}4.0-4.7 \\
8.3-9.8 \\
30.5-33.3 \\
20.6-24.4 \\
49.4-55.7 \\
3.7-5.3\end{array}$ & $\begin{array}{r}4.2 \\
9.0 \\
32.4 \\
22.1 \\
52.4 \\
4.9\end{array}$ & $\begin{array}{l}6.1 \\
3.2 \\
1.9 \\
3.9 \\
2.1 \\
9.7\end{array}$ & $\begin{array}{c}3.6-4.8 \\
8.6-10.0 \\
31.3-33.7 \\
20.7-24.0 \\
49.6-54.2 \\
4.1-5.7\end{array}$ \\
\hline $\begin{array}{l}\mathrm{BcbH} \\
\mathrm{BcC} \\
\text { RstA }\end{array}$ & $\begin{array}{l}29.8 \\
19.1 \\
51.8\end{array}$ & $\begin{array}{l}3.3 \\
7.0 \\
2.9\end{array}$ & $\begin{array}{l}27.4-33.2 \\
15.9-22.2 \\
50.0-56.0\end{array}$ & $\begin{array}{l}30.0 \\
19.3 \\
52.3\end{array}$ & $\begin{array}{l}3.0 \\
6.9 \\
3.6\end{array}$ & $\begin{array}{l}28.4-32.2 \\
17.2-22.4 \\
48.0-56.0\end{array}$ & $\begin{array}{l}29.5 \\
18.4 \\
51.7\end{array}$ & $\begin{array}{l}4.1 \\
7.7 \\
3.5\end{array}$ & $\begin{array}{l}27.8-32.0 \\
16.1-20.8 \\
49.0-55.0\end{array}$ \\
\hline$\overline{\mathrm{x}} \mathrm{C} . \mathrm{V}$. & & 3.9 & & & 3.7 & & & 3.8 & \\
\hline
\end{tabular}

Table 2

Sample statistics of the 23 skull measurements performed in $M$. foina from Poland.

\begin{tabular}{|c|c|c|c|c|c|c|c|c|c|}
\hline & \multicolumn{9}{|c|}{ Females $(n=22)$} \\
\hline & \multicolumn{3}{|c|}{ Juveniles $(n=10)$} & \multicolumn{3}{|c|}{ Subadults $(n=5)$} & \multicolumn{3}{|c|}{ Adults ( $n=7)$} \\
\hline & $\overline{\mathbf{x}}$ & C. V. & O. R & $\overline{\overline{\mathbf{x}}}$ & C. V. & O. R & $\overline{\overline{\mathbf{x}}}$ & C. V. & O. $\mathrm{R}$ \\
\hline $\begin{array}{l}\text { CbL } \\
\text { PrL } \\
\text { FcL } \\
\text { BeL } \\
\text { CmL } \\
\text { MxtL } \\
\text { PtL }\end{array}$ & $\begin{array}{l}76.1 \\
79.3 \\
53.5 \\
49.9 \\
30.7 \\
27.3 \\
35.7\end{array}$ & $\begin{array}{l}2.0 \\
1.8 \\
3.0 \\
1.4 \\
3.7 \\
4.7 \\
3.2\end{array}$ & $\begin{array}{l}73.7-78.8 \\
77.7-82.1 \\
33.7-37.5 \\
48.9-51.1 \\
29.3-32.9 \\
26.0-30.4 \\
34.3-38.0\end{array}$ & $\begin{array}{l}77.8 \\
80.2 \\
36.5 \\
50.5 \\
31.3 \\
28.0 \\
37.0\end{array}$ & $\begin{array}{l}2.4 \\
2.9 \\
3.1 \\
5.0 \\
3.9 \\
5.6 \\
3.3\end{array}$ & $\begin{array}{l}75.2-79.7 \\
77.4-83.2 \\
35.2-37.8 \\
47.8-53.8 \\
30.5-33.2 \\
26.2-30.5 \\
35.3-38.2\end{array}$ & $\begin{array}{l}78.6 \\
81.9 \\
36.3 \\
51.8 \\
31.5 \\
28.2 \\
36.9\end{array}$ & $\begin{array}{l}1.3 \\
1.4 \\
1.9 \\
3.2 \\
0.7 \\
2.0 \\
2.3\end{array}$ & $\begin{array}{l}76.6-79.9 \\
80.1-83.5 \\
35.2-37.1 \\
49.8-54.7 \\
31.1-31.8 \\
27.3-29.0 \\
36.0-38.5\end{array}$ \\
\hline $\begin{array}{l}\text { ZyW } \\
\text { EorW } \\
\text { IorW } \\
\text { PorW } \\
\text { MstW } \\
\text { BmW } \\
\text { RtrW }\end{array}$ & $\begin{array}{l}45.2 \\
24.0 \\
20.2 \\
18.5 \\
36.1 \\
22.3 \\
16.3\end{array}$ & $\begin{array}{l}4.6 \\
6.3 \\
3.3 \\
2.8 \\
5.0 \\
4.3 \\
2.9\end{array}$ & $\begin{array}{l}41.8-47.6 \\
22.3--27.2 \\
19.2-21.3 \\
17.6-19.2 \\
31.6-38.4 \\
20.9-24.0 \\
15.6-17.1\end{array}$ & $\begin{array}{l}46.6 \\
25.1 \\
20.7 \\
20.1 \\
36.0 \\
22.8 \\
17.0\end{array}$ & $\begin{array}{l}1.4 \\
1.8 \\
1.4 \\
1.4 \\
6.7 \\
2.7 \\
4.7\end{array}$ & $\begin{array}{l}45.6-47.1 \\
24.4-25.6 \\
20.4-21.1 \\
19.9-20.3 \\
32.6-38.0 \\
22.2-23.5 \\
16.3-18.4\end{array}$ & $\begin{array}{l}48.1 \\
24.9 \\
20.7 \\
17.6 \\
37.0 \\
23.9 \\
16.7\end{array}$ & $\begin{array}{l}1.1 \\
9.4 \\
2.9 \\
4.2 \\
5.3 \\
5.6 \\
2.5\end{array}$ & $\begin{array}{l}47.2-48.8 \\
22.8-28.7 \\
20.0-21.8 \\
16.4-18.4 \\
33.3-39.3 \\
21.0-25.3 \\
15.8-17.1\end{array}$ \\
\hline $\begin{array}{l}\mathrm{LcL} \\
\mathrm{LmL} \\
\mathrm{MntL} \\
\text { MnbH } \\
\text { MnbL } \\
\text { MnbW }\end{array}$ & $\begin{array}{r}4.1 \\
9.1 \\
31.1 \\
22.9 \\
50.4 \\
4.7\end{array}$ & $\begin{array}{l}3.5 \\
4.5 \\
2.6 \\
3.9 \\
2.2 \\
7.7\end{array}$ & $\begin{array}{c}4.0-4.4 \\
8.7-9.9 \\
30.1-32.7 \\
21.6-24.2 \\
48.8-52.7 \\
4.1-5.3\end{array}$ & $\begin{array}{r}4.3 \\
9.2 \\
31.8 \\
23.5 \\
51.5 \\
5.2\end{array}$ & $\begin{array}{l}2.5 \\
3.8 \\
2.3 \\
2.3 \\
2.1 \\
1.5\end{array}$ & $\begin{array}{c}4.2-4.4 \\
8.8-9.7 \\
31.1-33.0 \\
23.0-24.3 \\
50.1-52.6 \\
5.1-5.3\end{array}$ & $\begin{array}{r}4.2 \\
9.1 \\
32.1 \\
23.4 \\
52.0 \\
5.4\end{array}$ & $\begin{array}{l}3.1 \\
2.1 \\
1.6 \\
2.4 \\
1.0 \\
3.6\end{array}$ & $\begin{array}{c}4.1-4.4 \\
8.9-9.4 \\
31.6-33.0 \\
22.6-24.2 \\
51.5-52.9 \\
5.1-5.6\end{array}$ \\
\hline $\begin{array}{l}\text { BcbH } \\
\text { BcC } \\
\text { RstA }\end{array}$ & $\begin{array}{l}29.1 \\
19.3 \\
59.0\end{array}$ & $\begin{array}{l}2.1 \\
7.8 \\
4.3\end{array}$ & $\begin{array}{l}27.9-29.8 \\
17.7-22.0 \\
57.0-65.0\end{array}$ & $\begin{array}{l}29.7 \\
20.5 \\
59.2\end{array}$ & $\begin{array}{r}3.3 \\
11.6 \\
3.6\end{array}$ & $\begin{array}{l}28.3-30.6 \\
17.3-22.6 \\
56.0-61.0\end{array}$ & $\begin{array}{l}29.7 \\
20.1 \\
59.7\end{array}$ & $\begin{array}{l}2.4 \\
5.7 \\
3.3\end{array}$ & $\begin{array}{l}28.5-30.4 \\
19.0-22.1 \\
57.0-62.0\end{array}$ \\
\hline $\mathrm{xC} . \mathrm{V}$. & & 3.7 & & & 3.5 & & & 3.0 & \\
\hline
\end{tabular}


of abbreviations) in $M$. martes from Poland, according, to sex and age groups. $\overline{\mathrm{X}}$ : mean, variation. To simplify the table, only number of skulls (in brackets) of each group is of skulls.

\begin{tabular}{|c|c|c|c|c|c|c|c|c|}
\hline \multicolumn{9}{|c|}{ Males $(n=133)$} \\
\hline \multicolumn{3}{|c|}{ Juveniles ( $\mathrm{n}=33$} & \multicolumn{3}{|c|}{ Subadults $(n=43)$} & \multicolumn{3}{|c|}{ Adults $(n=57)$} \\
\hline$\overline{\mathbf{x}}$ & C. V. & O. $\mathbf{R}$ & $\overline{\mathbf{x}}$ & C. V. & $0 . \bar{R}$ & $\cdot \overline{\mathbf{x}}$ & C. V. & O. R \\
\hline $\begin{array}{l}82.7 \\
84.5 \\
38.0 \\
53.5 \\
39.9 \\
30.0 \\
40.8\end{array}$ & $\begin{array}{l}2.3 \\
2.3 \\
3.3 \\
2.9 \\
3.2 \\
4.4 \\
3.1\end{array}$ & $\begin{array}{l}77.9-86.2 \\
79.4-87.6 \\
35.1-40.3 \\
50.0-56.6 \\
31.3-35.7 \\
27.1-34.8 \\
37.8-43.2\end{array}$ & $\begin{array}{l}84.2 \\
86.5 \\
38.6 \\
54.3 \\
34.7 \\
30.7 \\
41.6\end{array}$ & $\begin{array}{l}1.7 \\
1.6 \\
2.8 \\
2.5 \\
2.5 \\
4.5 \\
2.6\end{array}$ & $\begin{array}{l}80.9-87.7 \\
83.5-89.3 \\
36.3-40.6 \\
51.3-57.1 \\
32.8-36.9 \\
28.7-35.7 \\
38.7-43.5\end{array}$ & $\begin{array}{l}85.6 \\
88.6 \\
39.3 \\
55.4 \\
35.3 \\
31.1 \\
42.3\end{array}$ & $\begin{array}{l}1.7 \\
1.7 \\
2.7 \\
2.5 \\
2.7 \\
2.9 \\
2.9\end{array}$ & $\begin{array}{l}82.0-87.9 \\
85.5-92.3 \\
36.6-41.8 \\
51.6-60.0 \\
33.5-38.7 \\
29.1-35.2 \\
40.0-45.0\end{array}$ \\
\hline $\begin{array}{l}46.4 \\
23.4 \\
20.0 \\
19.7 \\
38.8 \\
22.5 \\
17.0\end{array}$ & $\begin{array}{l}3.4 \\
4.4 \\
4.5 \\
7.0 \\
3.0 \\
3.9 \\
3.7\end{array}$ & $\begin{array}{l}41.9-48.8 \\
21.5-25.5 \\
18.7-22.7 \\
16.9-22.7 \\
36.3-41.0 \\
21.2-24.3 \\
15.5-18.0\end{array}$ & $\begin{array}{l}47.9 \\
24.2 \\
20.7 \\
19.5 \\
39.6 \\
23.3 \\
17.2\end{array}$ & $\begin{array}{l}2.8 \\
4.8 \\
3.9 \\
7.1 \\
2.7 \\
3.3 \\
2.3\end{array}$ & $\begin{array}{l}42.4-50.1 \\
21.7-28.6 \\
19.2-23.4 \\
15.6-21.9 \\
36.7-42.0 \\
21.3-24.9 \\
16.4-18.1\end{array}$ & $\begin{array}{l}51.1 \\
25.9 \\
21.5 \\
19.1 \\
40.6 \\
23.8 \\
17.7\end{array}$ & $\begin{array}{l}3.2 \\
5.3 \\
3.1 \\
6.5 \\
2.4 \\
3.5 \\
2.7\end{array}$ & $\begin{array}{l}47.6-55.2 \\
23.2-29.9 \\
19.6-22.9 \\
15.8-21.3 \\
38.0-43.0 \\
21.8-25.8 \\
16.6-18.7\end{array}$ \\
\hline $\begin{array}{r}4.8 \\
9.9 \\
34.5 \\
23.9 \\
55.2 \\
5.8\end{array}$ & $\begin{array}{l}4.9 \\
4.7 \\
2.0 \\
3.8 \\
3.1 \\
8.2\end{array}$ & $\begin{array}{c}4.2-5.1 \\
9.0-11.2 \\
33.0-35.6 \\
22.2-26.1 \\
51.6-58.8 \\
5.0-6.8\end{array}$ & $\begin{array}{r}4.9 \\
10.0 \\
34.9 \\
24.3 \\
56.7 \\
6.4\end{array}$ & $\begin{array}{l}4.1 \\
3.6 \\
2.1 \\
3.5 \\
1.9 \\
6.3\end{array}$ & $\begin{array}{c}4.5-5.4 \\
9.2--11.0 \\
33.4-36.6 \\
22.6-26.3 \\
54.8-59.5 \\
5.6-7.6\end{array}$ & $\begin{array}{r}4.7 \\
9.9 \\
35.6 \\
25.0 \\
58.0 \\
7.2\end{array}$ & $\begin{array}{l}3.9 \\
2.8 \\
2.4 \\
3.6 \\
1.8 \\
8.6\end{array}$ & $\begin{array}{c}4.3-5.2 \\
9.1-10.6 \\
34.0-37.5 \\
22.5-27.1 \\
56.1-60.3 \\
5.5-8.5\end{array}$ \\
\hline $\begin{array}{l}31.7 \\
22.9 \\
50.5\end{array}$ & $\begin{array}{l}2.6 \\
7.2 \\
3.5\end{array}$ & $\begin{array}{l}30.1-33.2 \\
18.9-24.8 \\
47.0-54.0\end{array}$ & $\begin{array}{l}31.9 \\
22.7 \\
51.0\end{array}$ & $\begin{array}{l}2.4 \\
7.3 \\
3.2\end{array}$ & $\begin{array}{r}30.4-33.9 \\
19.0 \ldots 26.0 \\
47.0\end{array}$ & $\begin{array}{l}32.4 \\
22.6 \\
51.7\end{array}$ & $\begin{array}{l}3.2 \\
7.8 \\
3.9\end{array}$ & $\begin{array}{l}29.7-35.9 \\
18.1-25.8 \\
47.0-56.0\end{array}$ \\
\hline & 4.0 & & & 3.5 & & & 3.6 & \\
\hline
\end{tabular}

See Table 1 for explanation of symbols.

\begin{tabular}{|c|c|c|c|c|c|c|c|c|}
\hline \multicolumn{9}{|c|}{ Males $(n=25)$} \\
\hline \multicolumn{3}{|c|}{ Juveniles $(n=4)$} & \multicolumn{3}{|c|}{ Subadults $(n=9)$} & \multicolumn{3}{|c|}{ Adults $(n=12)$} \\
\hline$\overline{\mathbf{x}}$ & C. V. & O. R & $\overline{\overline{\mathbf{x}}}$ & C. V. & O.R & $\dot{\mathbf{x}}$ & C. V. & O. R \\
\hline $\begin{array}{l}80.4 \\
83.4 \\
37.2 \\
52.8 \\
32.8 \\
29.1 \\
38.8\end{array}$ & $\begin{array}{l}1.5 \\
1.3 \\
1.4 \\
1.9 \\
3.3 \\
2.6 \\
2.8\end{array}$ & $\begin{array}{l}78.7-81.4 \\
82.3-84.7 \\
36.8-38.0 \\
51.8-54.1 \\
31.8-34.3 \\
28.4-30.1 \\
37.6-40.0\end{array}$ & $\begin{array}{l}81.1 \\
85.3 \\
38.1 \\
54.0 \\
33.4 \\
29.2 \\
38.4\end{array}$ & $\begin{array}{l}1.7 \\
1.7 \\
1.7 \\
1.7 \\
2.9 \\
2.8 \\
1.6\end{array}$ & $\begin{array}{l}78.2-82.3 \\
82.6-86.7 \\
\mathbf{3 6 . 7}-38.9 \\
\mathbf{5 2 . 4 - 5 5 . 2} \\
\mathbf{3 1 . 7}-\mathbf{3 4 . 6} \\
28.2-\mathbf{3 0 . 7} \\
37.4--39.2\end{array}$ & $\begin{array}{l}82.4 \\
86.7 \\
38.5 \\
55.6 \\
34.5 \\
29.7 \\
39.6\end{array}$ & $\begin{array}{l}1.6 \\
1.6 \\
1.8 \\
2.2 \\
2.4 \\
2.3 \\
2.3\end{array}$ & $\begin{array}{l}80.7-84.9 \\
83.6-88.3 \\
37.6-40.0 \\
54.4-57.9 \\
33.0-35.6 \\
28.7-30.9 \\
38.2-40.9\end{array}$ \\
\hline $\begin{array}{l}47.8 \\
25.8 \\
21.5 \\
17.5 \\
37.7 \\
24.3 \\
17.3\end{array}$ & $\begin{array}{l}3.1 \\
2.1 \\
4.9 \\
4.6 \\
2.3 \\
2.4 \\
2.8\end{array}$ & $\begin{array}{l}46.1-49.4 \\
25.3-26.4 \\
20.7-23.0 \\
16.6-18.0 \\
36.8-38.7 \\
23.7-25.0 \\
16.7-17.9\end{array}$ & $\begin{array}{l}49.3 \\
26.3 \\
21.6 \\
20.0 \\
38.4 \\
24.8 \\
17.6\end{array}$ & $\begin{array}{l}2.7 \\
2.9 \\
2.9 \\
4.5 \\
2.4 \\
3.7 \\
3.6\end{array}$ & $\begin{array}{l}47.3-51.5 \\
25.2-27.2 \\
21.1-23.2 \\
18.5-21.1 \\
36.3-39.5 \\
23.5-26.3 \\
16.8-18.5\end{array}$ & $\begin{array}{l}52.3 \\
28.2 \\
22.9 \\
19.8 \\
39.6 \\
24.8 \\
18.3\end{array}$ & $\begin{array}{l}2.9 \\
4.0 \\
3.6 \\
6.1 \\
2.6 \\
3.6 \\
1.6\end{array}$ & $\begin{array}{l}51.0-55.6 \\
26.1-30.0 \\
22.0-24.2 \\
18.3-21.5 \\
37.8-41.4 \\
23.8-26.4 \\
17.9-18.8\end{array}$ \\
\hline $\begin{array}{r}4.7 \\
9.8 \\
33.7 \\
25.0 \\
54.0 \\
5.9\end{array}$ & $\begin{array}{l}3.7 \\
5.5 \\
1.8 \\
5.9 \\
1.5 \\
7.6\end{array}$ & $\begin{array}{c}4.4 \cdots 4.8 \\
9.0-10.3 \\
33.1-34.6 \\
23.7-27.2 \\
53.3-55.0 \\
5.2-6.3\end{array}$ & $\begin{array}{r}4.7 \\
9.7 \\
33.4 \\
25.1 \\
54.8 \\
6.5\end{array}$ & $\begin{array}{l}3.8 \\
4.6 \\
1.6 \\
2.6 \\
1.7 \\
3.8\end{array}$ & $\begin{array}{c}4.4-4.9 \\
8.7-10.2 \\
32.7-34.3 \\
24.4-26.4 \\
52.9-55.9 \\
6.1-6.9\end{array}$ & $\begin{array}{r}4.8 \\
10.0 \\
34.1 \\
26.6 \\
55.9 \\
7.5\end{array}$ & $\begin{array}{l}3.2 \\
4.3 \\
2.8 \\
2.2 \\
1.7 \\
6.5\end{array}$ & $\begin{array}{c}4.5-4.9 \\
9.3-10.9 \\
32.6-35.9 \\
25.7-27.7 \\
54.7-57.7 \\
6.7-8.3\end{array}$ \\
\hline \multirow[t]{2}{*}{$\begin{array}{l}30.3 \\
21.1 \\
57.2\end{array}$} & $\begin{array}{l}2.8 \\
2.2 \\
3.8\end{array}$ & $\begin{array}{l}29.3-31.2 \\
20.6-21.7 \\
55.0-60.0\end{array}$ & $\begin{array}{l}31.2 \\
23.2 \\
58.7\end{array}$ & $\begin{array}{l}2.4 \\
7.2 \\
2.3\end{array}$ & $\begin{array}{l}30.4-32.5 \\
22.2-25.0 \\
57.0-60.0\end{array}$ & $\begin{array}{l}31.8 \\
22.2 \\
60.6\end{array}$ & $\begin{array}{l}1.4 \\
7.5 \\
4.3\end{array}$ & $\begin{array}{l}31.2-32.5 \\
20.0-24.7 \\
58.0-65.0\end{array}$ \\
\hline & 3.1 & & & 2.9 & & & 3.2 & \\
\hline
\end{tabular}




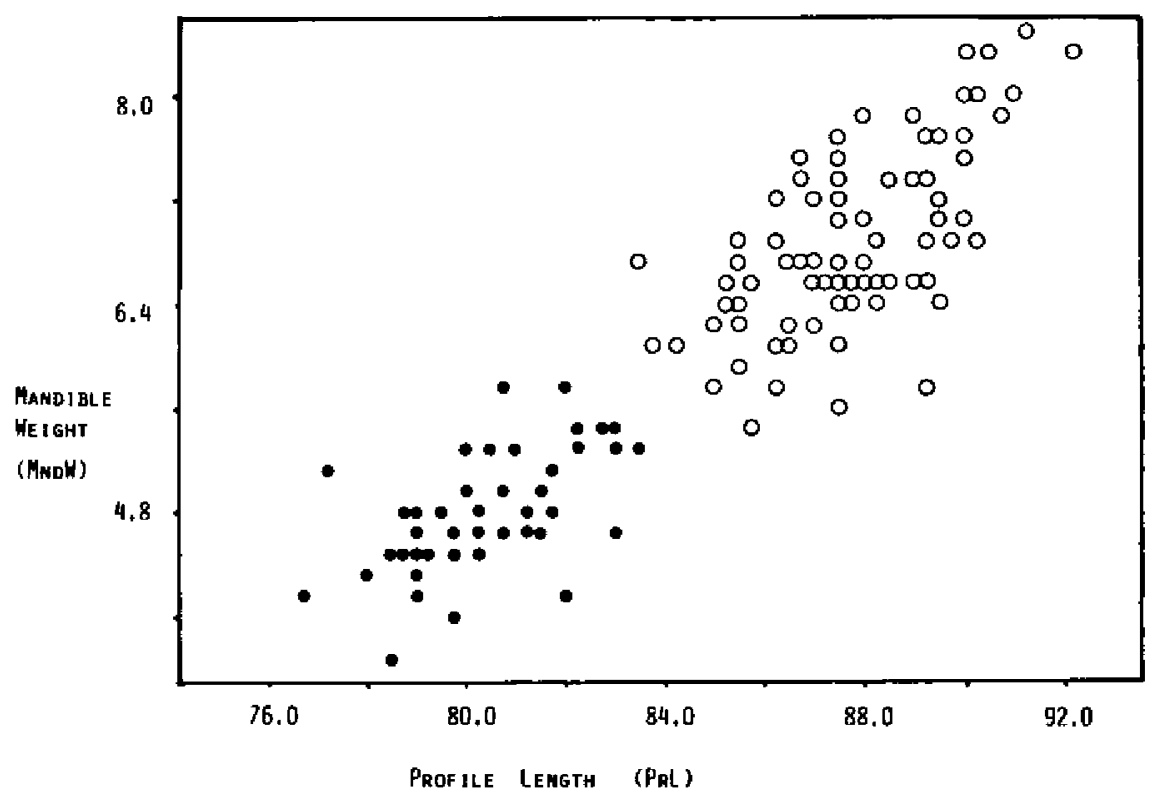

Fig. 2. Plot of Mandible Weight (g) against Profile Lenght (mm) to show the separation of males (open circle) and females (solid circle) in $M$. martes. Adult and subadult pooled.

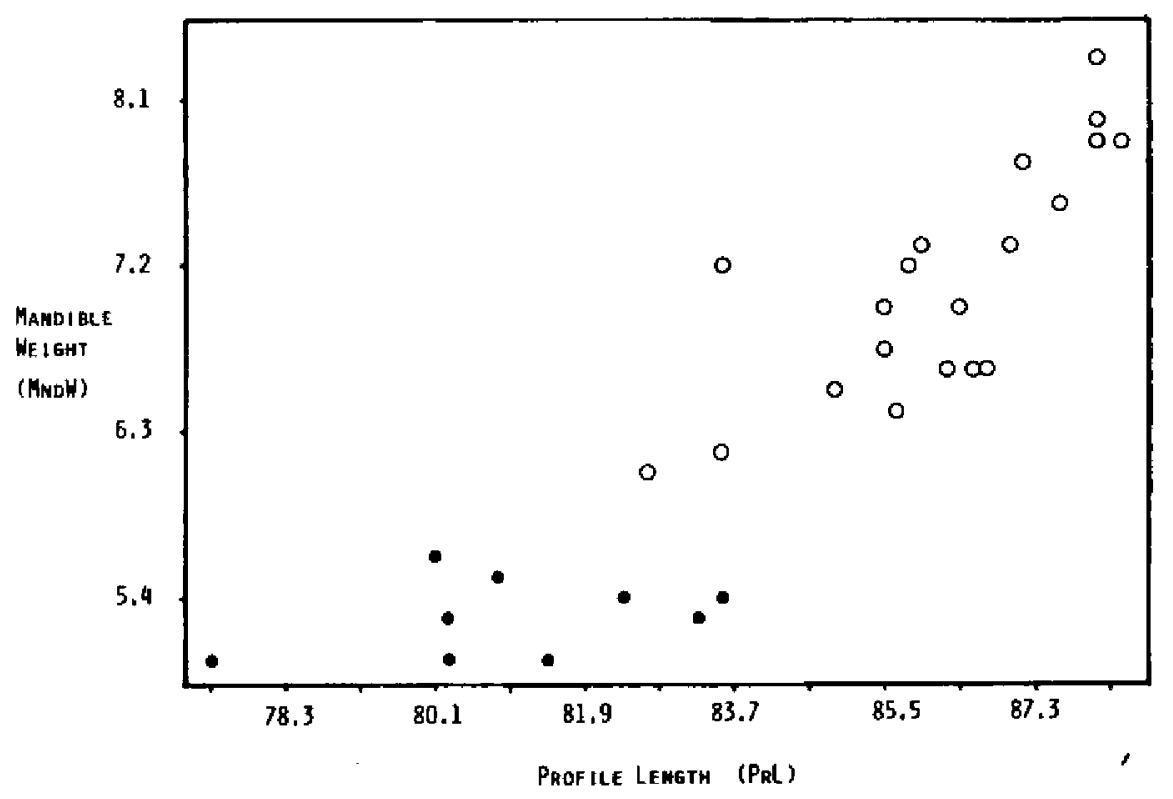

Fig. 3. Plot of the Mandible Weight (g) against Profile Lenght (mm) to show the separation of males (open circle) and females (solid circle) in M. martes. Adult and subadult pooled. 


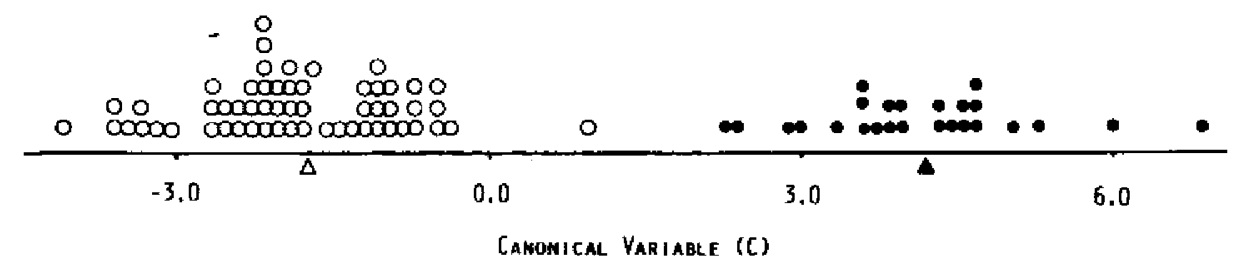

$[=133.64-1.03($ CBL $)-0.99(\mathrm{MmOW})-0.59$ (7YW)

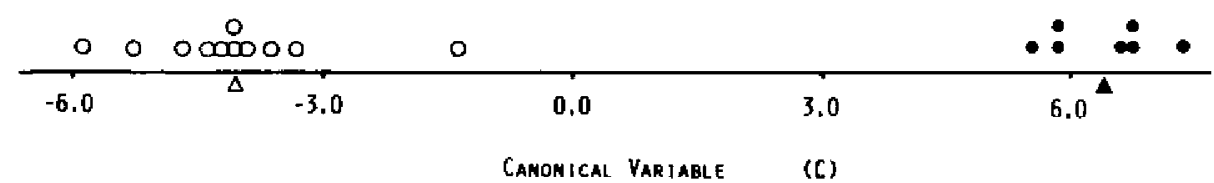

Fig. 4. Histogram of canonical variable score of adult male (open circle) and female (solid circle) of. $M$. martes (upper) and $M$. foina (lower). On the top of each histogram, coefficient of the measurements included in the canonical variable that separates males and females. Triangles indicate group centroids.

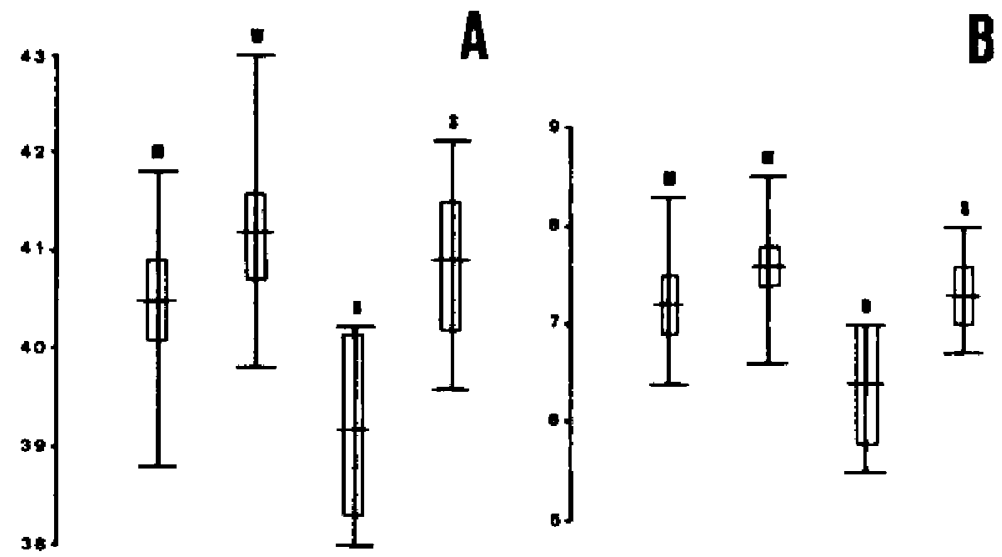

Fig. 5. Observed rangers, means, and $95 \%$ confidence intervals of and mastoid width (mm): A; mandible weight $(\mathrm{g})$ : B; of adult males of $M$. martes from 4 Polish populations. Pomerania and Masurian Lake Region; W: Wielkopolska-Kujawy Lowlands; B: Białowieża Primaeval Forest; S: Lower and Upper Silesia. 
Stepwise discriminant analysis provided a set of three traits, out of the 23 , being sufficient to separate males and females maximally, explaining $100 \%$ of the total variance. In $M$. martes these three measurements were $\mathrm{ZyW}, \mathrm{CmL}$ and PtL, all of them with similar contributions to the canonical variable (Fig. 4). In $M$. foina, the same analysis resulted in a combination of $\mathrm{CbL}, \mathrm{ZyW}$ and $\mathrm{MndW}$, both $\mathrm{CbL}$ and MndW showing high contributions into the canonical variable (Fig. 4). Classification of specimens following the DF was $100 \%$ correct in both species.

\subsection{Age Variation}

Although the number of age groups was small for an analysis of ontogenic process, the three groups considered allowed a general view of the characters and regions of the skull which exhibited variation connected with age.

Table 3

Results of ANOVA between adult males and females of each species in the 23 skull measurements and three indices. $F$ values were highly significant $(p<0.001)$ in all traits, except RstA (not significant). d.f: degrees of freedom. \%D: percentage of difference between means in both sexes $[(\overline{\mathbf{x}}$ male $-\overline{\mathbf{x}}$ female $) / \overline{\mathbf{x}}$ female $\times 100] . \bar{X}_{\% \mathrm{p}}$ : average of $\% \mathrm{D}$ in the 23 measurements.

\begin{tabular}{|c|c|c|c|c|}
\hline \multirow{2}{*}{ Meas./Indx. } & \multicolumn{2}{|c|}{ M. martes } & \multicolumn{2}{|c|}{$M$. foina } \\
\hline & $\% \mathrm{D}$ & $F^{l}$ & $\% D$ & $F^{2}$ \\
\hline $\begin{array}{l}\text { CbL } \\
\text { PrL } \\
\text { FcL } \\
\text { BcL } \\
\text { CmL } \\
\text { MxtL } \\
\text { PtI }\end{array}$ & $\begin{array}{r}8.7 \\
9.4 \\
8.6 \\
8.9 \\
11.9 \\
11.4 \\
10.7\end{array}$ & $\begin{array}{l}364.6 \\
380.7 \\
142.4 \\
177.6 \\
282.6 \\
160.6 \\
251.9\end{array}$ & $\begin{array}{l}4.8 \\
5.9 \\
6.1 \\
7.3 \\
9.5 \\
4.9 \\
7.3\end{array}$ & $\begin{array}{l}39.2 \\
54.5 \\
49.3 \\
25.3 \\
88.0 \\
25.1 \\
35.7\end{array}$ \\
\hline $\begin{array}{l}\text { ZyW } \\
\text { EorW } \\
\text { lorW } \\
\text { PorW } \\
\text { MstW } \\
\text { BmW } \\
\text { RtrW }\end{array}$ & $\begin{array}{r}12.9 \\
13.2 \\
10.9 \\
10.5 \\
8.9 \\
7.6 \\
11.4\end{array}$ & $\begin{array}{r}327.0 \\
135.2 \\
132.1 \\
35.7 \\
173.5 \\
54.4 \\
266.1\end{array}$ & $\begin{array}{r}8.7 \\
13.3 \\
10.6 \\
12.5 \\
7.0 \\
3.8 \\
9.6\end{array}$ & $\begin{array}{r}50.2 \\
24.0 \\
44.1 \\
23.4 \\
13.6 \\
2.8 \\
106.6\end{array}$ \\
\hline $\begin{array}{l}\text { LcL } \\
\text { LmL, } \\
\text { MntL } \\
\text { MnbH } \\
\text { MnbL } \\
\text { MnbW }\end{array}$ & $\begin{array}{r}10.2 \\
9.1 \\
9.9 \\
13.2 \\
10.7 \\
46.7\end{array}$ & $\begin{array}{l}117.4 \\
123.2 \\
346.5 \\
178.0 \\
438.7 \\
267.2\end{array}$ & $\begin{array}{r}14.3 \\
9.3 \\
6.2 \\
13.7 \\
7.5 \\
38.9\end{array}$ & $\begin{array}{r}45.6 \\
17.7 \\
25.4 \\
123.6 \\
97.9 \\
105.3\end{array}$ \\
\hline $\begin{array}{l}\text { BcbH } \\
\text { BcC } \\
\text { RstA }\end{array}$ & $\begin{array}{c}10.1 \\
22.9 \\
0.02\end{array}$ & $\begin{array}{c}120.5 \\
84.6 \\
\text { (ns) }\end{array}$ & $\begin{array}{r}7.1 \\
10.4 \\
1.5\end{array}$ & $\begin{array}{l}58.3 \\
10.8 \\
\text { (ns) }\end{array}$ \\
\hline$\overline{\mathrm{X}}_{\% \mathrm{D}}$ & 12.1 & & 9.5 & \\
\hline $\begin{array}{l}\text { MndW/MndL } \\
\text { MndW/BcbH } \\
\text { MndW/Prl }\end{array}$ & $\begin{array}{l}32.4 \\
33.2 \\
34.1\end{array}$ & $\begin{array}{l}191.1 \\
208.0 \\
194.5\end{array}$ & $\begin{array}{l}24.9 \\
26.7 \\
25.8\end{array}$ & $\begin{array}{r}65.2 \\
119.7 \\
82.2\end{array}$ \\
\hline
\end{tabular}

${ }^{1} \mathrm{df}=1,80 ; \quad 2 \mathrm{df}=1,17$. 
Four different ANOVA test were made among the three age classes, separating skulls by sex and species. Results indicated that MndW, MndL and $\mathrm{ZyW}$ are the measurements with maximum significance for age variation in both species (Tables 4,5 ). Other mandible traits also showed highly significant differences, which accounts for the continuous mandibular growth found by Pavlinov (1977) in $M$. martes. In $M$. foina, $F$ values were very low, especially in females. This reduced significance, rather than denote less age variation, might be explained as an artifact connected with the reduced and uneven sample size, since values of $\% \mathrm{D}$ between juveniles and subadults were even higher than

Table 4

Differences between the three age classes considered in females and males of $M$. martes. \%D: percentage of difference between means of juveniles and adults $[(\bar{x}$ adult $-\bar{x}$ juvenile $) / \bar{x}$ juvenile $\times 100] . \bar{x} \% \mathrm{D}$ : average of $\% \mathrm{D}$ for each sex in the 23 measurements. $F$ : significance of the differences obtained by ANOVA between the three age groups. d.f.: degrees of freedom Asterisks indicate the degree of significance of pairwise comparison of means by $t$-test between juvenile and subadult (Juv/Sba); subadult and adult (Sba/Ad).*** $p<0.001^{* *} p<0.01 * p<0.05 ;-$ not significant. All $F$ values were highly significant $(p<0.001)$, unless otherwise marked.

\begin{tabular}{|c|c|c|c|c|c|c|c|c|}
\hline \multirow{2}{*}{ Meas./Indx } & \multicolumn{2}{|c|}{$\% \mathrm{D}$} & \multirow{2}{*}{$\begin{array}{c}\mathbf{F}^{\mathbf{l}} \\
\wp \nsubseteq\end{array}$} & \multirow{2}{*}{$\begin{array}{c}\mathbf{F}^{2} \\
\sigma^{0} \sigma^{\prime}\end{array}$} & \multicolumn{2}{|c|}{ Juv/Sba } & \multicolumn{2}{|c|}{$\mathrm{Sba} / \mathrm{Ad}$} \\
\hline & 우 & $\sigma^{\prime \prime} \sigma^{\pi}$ & & & 99 & $\sigma^{\circ}$ & 우요 & $\sigma^{7} \sigma$ \\
\hline $\begin{array}{l}\text { CbL } \\
\text { PrL } \\
\text { FcL } \\
\text { BeL } \\
\text { CmL } \\
\text { MxtL } \\
\text { PtL }\end{array}$ & $\begin{array}{l}2.7 \\
3.0 \\
3.3 \\
2.3 \\
3.1 \\
2.4 \\
2.2\end{array}$ & $\begin{array}{l}3.5 \\
4.8 \\
3.4 \\
3.6 \\
4.2 \\
3.6 \\
3.6\end{array}$ & $\begin{array}{r}14.0 \\
14.7 \\
12.1 \\
7.4 \\
13.2 \\
6.7^{* *}\end{array}$ & $\begin{array}{r}32.0 \\
60.0 \\
12.4 \\
17.7 \\
21.5 \\
7.8 \\
14.7\end{array}$ & $\begin{array}{l}* * \\
* * \\
* * * \\
* * * \\
* \\
*\end{array}$ & $\begin{array}{l}* * * \\
* * * \\
* \\
* * * \\
* * \\
* *\end{array}$ & $\begin{array}{l}* \\
* * \\
* * * \\
- \\
-\end{array}$ & $\begin{array}{l}* * * \\
* * * \\
* * \\
* * * \\
* * \\
* \\
*\end{array}$ \\
\hline $\begin{array}{l}\text { Zyw } \\
\text { EorW } \\
\text { IorW } \\
\text { PorW } \\
\text { MstW } \\
\text { BmW } \\
\text { RtrW }\end{array}$ & $\begin{array}{r}4.3 \\
4.2 \\
3.9 \\
-6.3 \\
3.4 \\
4.0 \\
3.6\end{array}$ & $\begin{array}{r}10.2 \\
10.7 \\
7.4 \\
-2.9 \\
4.6 \\
5.7 \\
4.5\end{array}$ & $\begin{array}{r}20.2 \\
13.1 \\
8.7 \\
9.2 \\
10.3 \\
7.6 \\
7.7\end{array}$ & $\begin{array}{r}97.6 \\
41.8 \\
32.9 \\
28.9 \\
22.3 \\
27.5\end{array}$ & $\begin{array}{l}* * * \\
* * * \\
* * * \\
* \\
* \\
*\end{array}$ & $\begin{array}{l}* * * \\
* * \\
* * \\
* * * \\
-\end{array}$ & $\begin{array}{l}* \\
\bar{Z} \\
* * \\
- \\
-\end{array}$ & $\begin{array}{l}* * * \\
* * * \\
* * * \\
-* * \\
* * * \\
* * *\end{array}$ \\
\hline $\begin{array}{l}\text { LeL } \\
\text { LmL } \\
\text { MntL } \\
\text { MnbH } \\
\text { MnbL } \\
\text { MnbW }\end{array}$ & $\begin{array}{r}1.1 \\
0.8 \\
3.1 \\
3.5 \\
3.7 \\
13.6\end{array}$ & $\begin{array}{r}-1.0 \\
0.5 \\
3.2 \\
4.9 \\
5.0 \\
23.9\end{array}$ & $\begin{array}{r}- \\
15.5 \\
7.2 \\
22.9 \\
14.5\end{array}$ & $\begin{array}{r}-\overline{-} \\
21.5 \\
18.3 \\
46.9 \\
74.5\end{array}$ & $\begin{array}{l}\text { Z } \\
\bar{*} \\
* * \\
* * * \\
* * *\end{array}$ & $\begin{array}{l}- \\
* \\
* * * \\
* * *\end{array}$ & $\begin{array}{l}- \\
z \\
- \\
-\end{array}$ & $\begin{array}{l}\text { 二 } \\
\text { *** } \\
* * * \\
* * * \\
* * *\end{array}$ \\
\hline $\begin{array}{l}\text { BebH } \\
\text { BcC } \\
\text { RstA }\end{array}$ & $\begin{array}{l}-1.2 \\
-4.0 \\
-0.3\end{array}$ & $\begin{array}{r}2.2 \\
-1.3 \\
2.4\end{array}$ & E & $\begin{array}{l}6.4^{* *} \\
4.1^{*}\end{array}$ & - & - & + & ** \\
\hline$x_{\% \mathrm{D}}$ & 2.3 & 4.5 & & & & & & \\
\hline $\begin{array}{l}\text { PorW/ZyW } \\
\text { MndW/PorW } \\
\text { MndW/BcbH }\end{array}$ & $\begin{array}{r}-10.3 \\
20.9 \\
10.2\end{array}$ & $\begin{array}{l}-9.8 \\
28.6 \\
22.9\end{array}$ & $\begin{array}{l}20.8 \\
21.3 \\
21.7\end{array}$ & $\begin{array}{l}24.5 \\
68.7 \\
82.8\end{array}$ & $\bar{*}$ & $\begin{array}{l}* \\
* * * \\
* * *\end{array}$ & $\begin{array}{l}* * * \\
* * * \\
*\end{array}$ & $\begin{array}{l}* * * \\
* * * \\
* * *\end{array}$ \\
\hline
\end{tabular}

Idf $=2,100 ;{ }^{2}=2,130$. 
in $M$. martes (Tables 4,5 ). Width measurements exhibited higher $F$ values than length ones, while the only ones related to height of the skull had no significant differences except in males (Tables 4,5 ). Characters related to tooth size varied independently of age (Tables 4,5 ). Pairwise comparison between contiguous age groups revealed significant differences between subadults and adults, especially in male specimens (Tables 4,5 ), indicating that growth of the skull continues after the first year of age. Sexual differences were observed in the age variation of the characters. Males showed greater differences between juvenile and adults (\%D) and more significant differences ( $F$ values) among age classes. Differences between age class II and III were also more significant among males. Specific differences were observed in the growth of traits related to the braincase region of the skull. $\mathrm{BcC}$ decreases with age in $M$. martes and the opposite was found for $M$. foina, although $F$ values were not significant in the two species. BcL showed high significant age variation in males of $M$. foina,

Table 5

Differences between the three age classes considered in females and males of $M$. foina. See Table 4. for explanation of symbols.

\begin{tabular}{|c|c|c|c|c|c|c|c|c|}
\hline \multirow{2}{*}{ Meas./Indx } & \multicolumn{2}{|c|}{$\% \mathrm{D}$} & \multirow{2}{*}{$\begin{array}{c}F^{1} \\
90\end{array}$} & \multirow{2}{*}{$\begin{array}{c}F^{2} \\
\sigma^{n} \sigma^{\prime}\end{array}$} & \multicolumn{2}{|c|}{ Juv/Sba } & \multicolumn{2}{|c|}{$\mathrm{Sba} / \mathrm{Ad}$} \\
\hline & 우우 & $0^{7} \phi^{7}$ & & & 우 & $\overline{\sigma \sigma}$ & 99 & $\overline{\sigma \sigma}$ \\
\hline $\mathrm{CbL}$ & 3.3 & 2.5 & $6.0 * *$ & $4.2^{*}$ & - & - & - & $*$ \\
\hline PrL & 3.2 & 4.0 & $5.5 * *$ & $8.5^{* *}$ & - & $*$ & - & - \\
\hline FcL & 2.1 & 3.3 & - & $5.1^{*}$ & - & * & - & - \\
\hline $\mathrm{BcL}$ & 3.8 & 5.5 & - & 10.6 & - & - & - & $* *$ \\
\hline $\mathrm{CmL}$ & 2.4 & 5.1 & - & $5.5^{* *}$ & - & - & - & $*$ \\
\hline MxtL & 3.5 & 2.4 & - & - & - & - & - & - \\
\hline PtL & 3.5 & 1.9 & $3.7^{*}$ & $4.6^{*}$ & * & - & - & $* *$ \\
\hline Zyw & 6.3 & 9.5 & $7.0^{* *}$ & 17.7 & - & - & - & $* * *$ \\
\hline EorW & 3.7 & 9.4 & 二 & 13.1 & - & 二 & - & $\begin{array}{l}* * * \\
* *\end{array}$ \\
\hline $\begin{array}{l}\text { IorW } \\
\text { PorW }\end{array}$ & $\begin{array}{r}2.6 \\
-4.6\end{array}$ & $\begin{array}{r}6.9 \\
13.2\end{array}$ & $1 \overline{12.4}$ & $\begin{array}{l}7.5^{* *} \\
6.6^{* *}\end{array}$ & 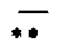 & $\overline{* *}$ & $\overline{* *}$ & $* *$ \\
\hline MstW & $\begin{array}{r}-4.0 \\
2.5\end{array}$ & $\begin{array}{r}13.2 \\
4.9\end{array}$ & 12.4 & $\begin{array}{l}0.0 \\
6.0^{* *}\end{array}$ & - & - & - & $*$ \\
\hline $\mathrm{BmW}$ & 7.2 & 2.1 & $4.6^{*}$ & - & - & - & - & - \\
\hline RtrW & 2.3 & 5.8 & - & $9.7^{* *}$ & * & - & - & ** \\
\hline LcL & 2.0 & 1.7 & - & - & - & - & - & - \\
\hline $\mathrm{LmL}$ & -0.3 & 1.7 & - & - & - & - & - & - \\
\hline MntL & 3.0 & 1.1 & - & - & - & - & - & \\
\hline MnbH & 2.1 & 6.3 & - & 11.3 & - & - & - & \\
\hline MnbL & 3.0 & 3.5 & $4.7^{*}$ & $7.3^{* *}$ & - & - & - & * \\
\hline MnbW & 15.4 & 27.6 & 12.2 & 29.2 & $* *$ & $*$ & - & $* * *$ \\
\hline $\mathrm{BcbH}$ & 2.2 & 4.8 & - & $7.0^{* *}$ & - & • & - & - \\
\hline $\mathrm{BcC}$ & 4.0 & 5.4 & - & - & - & - & - & - \\
\hline RstA & 1.2 & 5.9 & - & $4.0^{*}$ & - & - & - & - \\
\hline$X_{\% D}$ & 3.2 & 5.8 & & & & & & \\
\hline PorW/ZyW & -10.7 & 10.1 & 18.6 & 5.2 & - & $* *$ & $*$ & * \\
\hline Mndw/PorW & 24.1 & 9.0 & 16.5 & 8.6 & - & - & $* *$ & $* * *$ \\
\hline MndW/BcbH & 8.2 & 23.6 & 10.4 & 38.0 & $* *$ & $* *$ & - & $* * *$ \\
\hline
\end{tabular}

${ }^{1} \mathrm{df}=2,19 ;{ }^{2}=2 ; 22$. 
but very low in $M$. martes (Tables 4, 5). Finally, age variation in the height of the braincase was also very different in both species, $\% \mathrm{D}$ being much more larger in $M$. foina (Tables 4,5 ). Some skull proportions changed more significantly with age than single traits. Among them, $\mathrm{ZyW} / \mathrm{PorW}$, BcbH/MndW (Buchalczyk \& Ruprecht, 1977) and PrL/MndW, the last two being the most significant (Tables 4,5 ). These results suggest allometric growth of MndW and $\mathrm{ZyW}$, relative to the rest of measurements.

To investigate the association between measurements which synthesized distinct patterns of age variation, principal factor analysis (PFA) was performed in both species separately for males and females, using a data matrix in which skulls of the three age classes were pooled. In $M$. martes results were similar for males and females, the first three principal factors (PF) accounting for more than $87 \%$ of the total variance (Tables 6, 7). PF I and PF II clearly separates the measurements in length and width, respectively, while PF III joins those related to the braincase (Tables 6,7 ). This third factor is characterized by negative loadings from various measurements indicating shape variation. $\mathrm{BmW}$ and tooth measurements showed low loadings in the three PF. In $M$. foina four PF were obtained, results being different for males and females. In males the first three PF related width, length, and braincase measurements, respectively, while PF IV had only positive loadings from PorW (Table 8). In comparison with $M$. martes, the results found in $M$. foina differ in the relative significance of each PF. In the latter species, length and width factors showed similar contributions to the total variance explained (Table 8), while in $M$. martes, the length factor explained two times more variance than the width one (Table 6). PFA of females in $M$. foina resulted in non-meaningful factors, the first four PF only explaining $84.1 \%$ of the total variance (Table 9 ). This result must be related to the low $F$ values obtained for age variation and the small sample size metioned above.

\subsection{Inter-populaton Variation in $\boldsymbol{M}$. martes}

Eleven measurements exhibited signficant differences between populations of adult males of $M$. martes. The results of ANOVA of the four samples considered showed that width and height of the skull show the highest significant differences (Table 10), MndW and MstW exhibiting the highest $F$ values (Fig. 5).

Pairwise comparison of the four populations indicates that the results found by ANOVA are mainly due to differences between the Białowieża population and the other three. Wielkopolska and Silesia samples exhibited the most significant differences form the Bialowieża one, skulls from the latter population being the smallest of the whole 
Polish territory. Table 11 shows the values of $\mathrm{CbL}$ in the four populations of M. martes.

\subsection{Species Differentiation}

Although general appearance of the skull is distinctive in both species (Anderson, 1970), accurate identification based on skull measurements alone needs special indices (Altuna, 1973) or multivariate methods (Gerasimov, 1985), since measurements overlap widely (Tables 1, 2). Generally speaking, in the material examined, the skull of $M$. martes is more elongated, narrower and more flattened than that of $M$. foina.

Table 6

Rotated factor loadings of the first three principal factors (PF) derived from 23 skull measurements of males of $M$. martes from the three age classes pooled. These factors account for $86.9 \%$ of the total variance. VE: percentage of variance explained by each factor. Measurements have been rearranged so that the columns appear in decreasing order of variance explained by factors. Rows have also been rearranged so that for each successive factor, loadings greater than 0.500 appear first (Dixon, 1987). To facilitate the identification of the measurements associated in each factor boldface was used to show those measurements having highest loadings in a particular factor.

\begin{tabular}{lrrr}
\hline Meas. & PF I & \multicolumn{1}{c}{ PF II } & \multicolumn{1}{c}{ PF II } \\
\hline CbL & $\mathbf{0 . 9 0 6}$ & 0.255 & 0.045 \\
PrL & $\mathbf{0 . 8 8 5}$ & 0.238 & 0.002 \\
MndL & $\mathbf{0 . 8 5 8}$ & 0.161 & -0.020 \\
PtL & $\mathbf{0 . 8 5 3}$ & -0.018 & 0.175 \\
MntL & $\mathbf{0 . 8 1 3}$ & 0.211 & 0.039 \\
CmL & $\mathbf{0 . 8 0 3}$ & $\mathbf{0 . 1 5 6}$ & 0.053 \\
Fcl & $\mathbf{0 . 7 4 6}$ & $\mathbf{0 . 2 2 3}$ & -0.064 \\
MndW & $\mathbf{0 . 7 3 6}$ & 0.452 & -0.018 \\
RstW & $\mathbf{0 . 6 6 6}$ & 0.277 & 0.063 \\
MndH & $\mathbf{0 . 6 4 2}$ & 0.387 & 0.252 \\
BcL & $\mathbf{0 . 5 7 9}$ & $\mathbf{0 . 1 8 1}$ & -0.028 \\
MxtL & $\mathbf{0 . 5 5 3}$ & 0.071 & 0.037 \\
EorW & 0.343 & $\mathbf{0 . 7 3 2}$ & 0.007 \\
ZyW & 0.560 & $\mathbf{0 . 6 6 0}$ & 0.039 \\
MstW & 0.534 & $\mathbf{0 . 6 2 6}$ & 0.040 \\
RstA & -0.239 & $\mathbf{0 . 6 2 5}$ & 0.126 \\
IorW & 0.434 & $\mathbf{0 . 6 2 3}$ & -0.003 \\
BcbH & 0.270 & -0.018 & $\mathbf{0 . 9 0 1}$ \\
BcC & 0.120 & -0.005 & $\mathbf{0 . 8 1 6}$ \\
PorW & -0.162 & 0.192 & $\mathbf{0 . 7 8 8}$ \\
LmL & 0.364 & $\mathbf{0 . 0 1 6}$ & $\mathbf{0 . 0 8 9}$ \\
BmW & 0.359 & 0.403 & 0.058 \\
LeL & 0.306 & 0.136 & 0.144 \\
VE & $\mathbf{5 1 . 3 \%}$ & $\mathbf{1 8 . 9 \%}$ & $\mathbf{1 6 . 7 \%}$ \\
\hline
\end{tabular}


An ANOVA was performed separately for each sex, pooling together adult and subadult specimens. This pooling of age groups helped to solve the problem of small sample size in $M$. foina compared to $M$. martes.

With the exception of $\mathrm{BcC}$, all measurements showed significant differences, in one or both sexes (Table 12). RstA was the character with highest $F$, being the only one with no overlap between species when groups of the same sex and age are compared (Tables 1, 2, Fig. 6). Two character ratios showed large differences among species. These ratios were relating length and width of the skull: RstA/PtL and RstA/CbL (Table 12). Plotting of PtL against RstA allowed clear separation of species in both sexes (Figs. 7, 8). This difference between species in PtL must be related to the shape of the posterior edge of palatine which is usually more developed in $M$. martes (Vericad, 1970).

Specific differences were found to be different in males and females,

Table 7

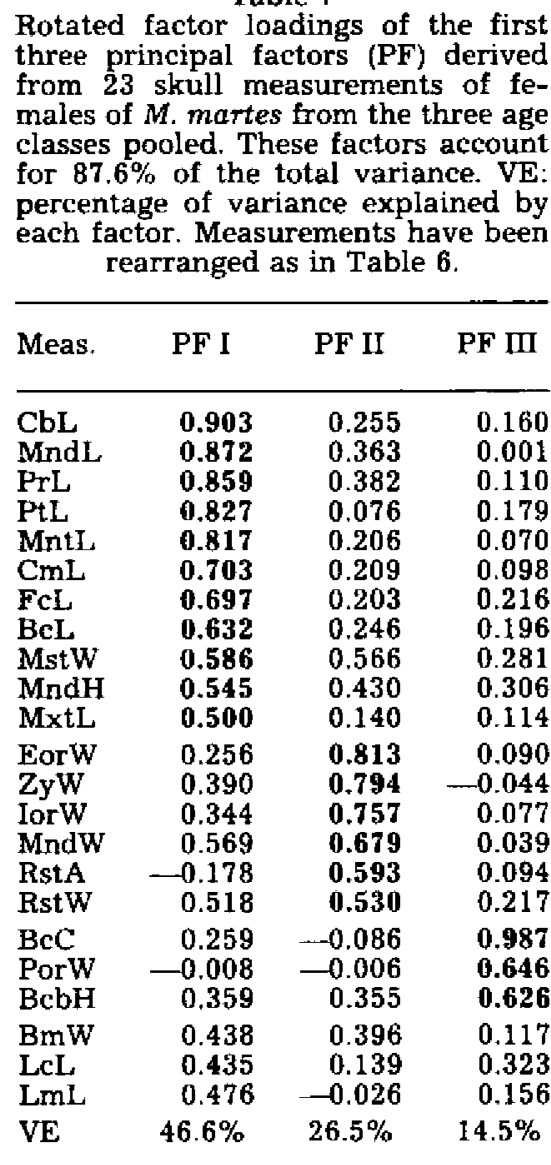


some characters exhibiting significant differences only in females, and vice versa. Eight traits, out of the 23 , had significant differences in both males and females, eleven had significant $F$ only in males, and three only in females. Males had higher $F$ values in the group of length measurements, PtL and $\mathrm{CbL}$ being the most different between both species (Table 12). However, the opposite was found in females, in which $\mathrm{ZyW}$ and $\mathrm{RtrW}$ had the highest $F$ values (Table 12). Sexual differences were found also in the relative size differences $(\% \mathrm{D})$ between the species. Females of $M$. foina are, on the average, $3.2 \%$ larger than. $M$. martes (Table 12). However, in males the average of $\% \mathrm{D}$ was close to zero, although differences were more apparent than in females, exhibiting an opposite trend between length and width measurements. $M$. martes is longer, while $M$. foina is larger in width (Table 12). The largest \% D were found in RstA, EorW, BmW, and MndW.

Accepting that the rostral angle is the best discriminating metrical character, a dividing point was determined for separation of the spe-

Table 8

Rotated factor loadings of the first four principal factors (PF) derived from 23 skull measurements of males of $M$. foina from the three age classes pooled. These factors account for $85.6 \%$ of the total variance. VE: percentage of variance explained by each factor. Measurements have been rearranged as in Table 6 .

\begin{tabular}{|c|c|c|c|c|}
\hline Meas. & PF I & PF II & PF III & PF rV \\
\hline $\begin{array}{l}\text { IorW } \\
\text { ZyW } \\
\text { EorW } \\
\text { RtrW } \\
\text { MstW } \\
\text { MnbW } \\
\text { RstA } \\
\text { MnbH } \\
\text { Bel }\end{array}$ & $\begin{array}{l}0.818 \\
0.804 \\
0.801 \\
0.761 \\
0.719 \\
0.716 \\
0.668 \\
0.604 \\
0.601\end{array}$ & $\begin{array}{c}0.079 \\
0.207 \\
0.129 \\
0.253 \\
0.139 \\
0.451 \\
-0.241 \\
0.278 \\
0.337\end{array}$ & $\begin{array}{r}0.066 \\
0.342 \\
0.057 \\
-0.022 \\
0.034 \\
0.282 \\
0.443 \\
0.225 \\
0.484\end{array}$ & $\begin{array}{r}0.091 \\
0.091 \\
0.263 \\
0.074 \\
0.040 \\
0.277 \\
-0.100 \\
0.039 \\
0.112\end{array}$ \\
\hline $\begin{array}{l}\text { MntL } \\
\text { MxtL } \\
\text { MnbL } \\
\text { PtL } \\
\text { LmL } \\
\text { CbL } \\
\text { CmL } \\
\text { PrL }\end{array}$ & $\begin{array}{r}0.006 \\
0.074 \\
0.455 \\
0.395 \\
-0.071 \\
0.439 \\
0.412 \\
0.488\end{array}$ & $\begin{array}{l}0.899 \\
0.819 \\
0.796 \\
0.774 \\
0.719 \\
0.714 \\
0.683 \\
0.644\end{array}$ & $\begin{array}{r}-0.179 \\
0.404 \\
0.061 \\
-0.095 \\
0.282 \\
0.023 \\
0.265 \\
0.316\end{array}$ & $\begin{array}{r}0.219 \\
0.036 \\
0.301 \\
-0.211 \\
-0.016 \\
0.107 \\
0.213 \\
0.295\end{array}$ \\
\hline $\begin{array}{l}\mathrm{BmW} \\
\mathrm{BcC} \\
\mathrm{BcbH}\end{array}$ & $\begin{array}{l}0.083 \\
0.049 \\
0.447\end{array}$ & $\begin{array}{r}0.072 \\
-0.138 \\
0.365\end{array}$ & $\begin{array}{l}0.762 \\
0.700 \\
0.578\end{array}$ & $\begin{array}{r}-0.016 \\
0.342 \\
0.339\end{array}$ \\
\hline PorW & 0.157 & 0.134 & 0.164 & 0.841 \\
\hline $\begin{array}{l}\text { LcL } \\
\text { FcL }\end{array}$ & $\begin{array}{l}0.074 \\
0.268\end{array}$ & $\begin{array}{l}0.103 \\
0.457\end{array}$ & $\begin{array}{l}0.421 \\
0.156\end{array}$ & $\begin{array}{r}-0.010 \\
0.445\end{array}$ \\
\hline $\mathrm{VE}$ & $31.7 \%$ & $29.9 \%$ & $16.7 \%$ & $9.3 \%$ \\
\hline
\end{tabular}




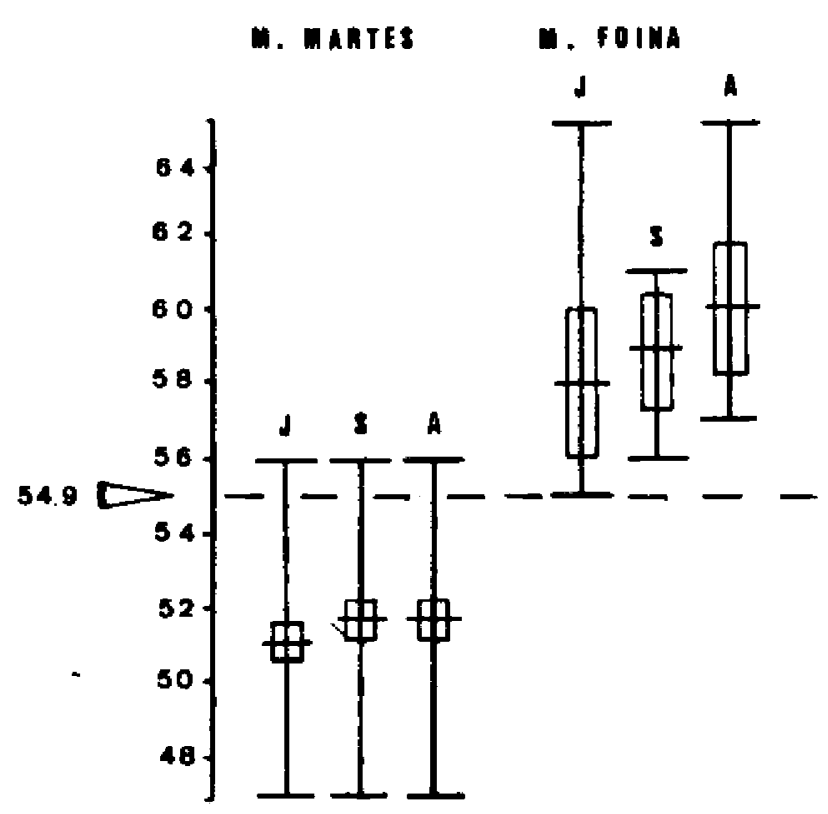

Fig. 6. Observed ranges, means, and $99 \%$ confidence intervals of rostral angle $\left({ }^{\circ}\right)$ in $M$. martes and $M$. foina, of the three age classes (males and females pooled). J: juveniles, $S$ : subadult, A: adult. Dividing point of $54.9^{\circ}$ is marked, see the text for further details.

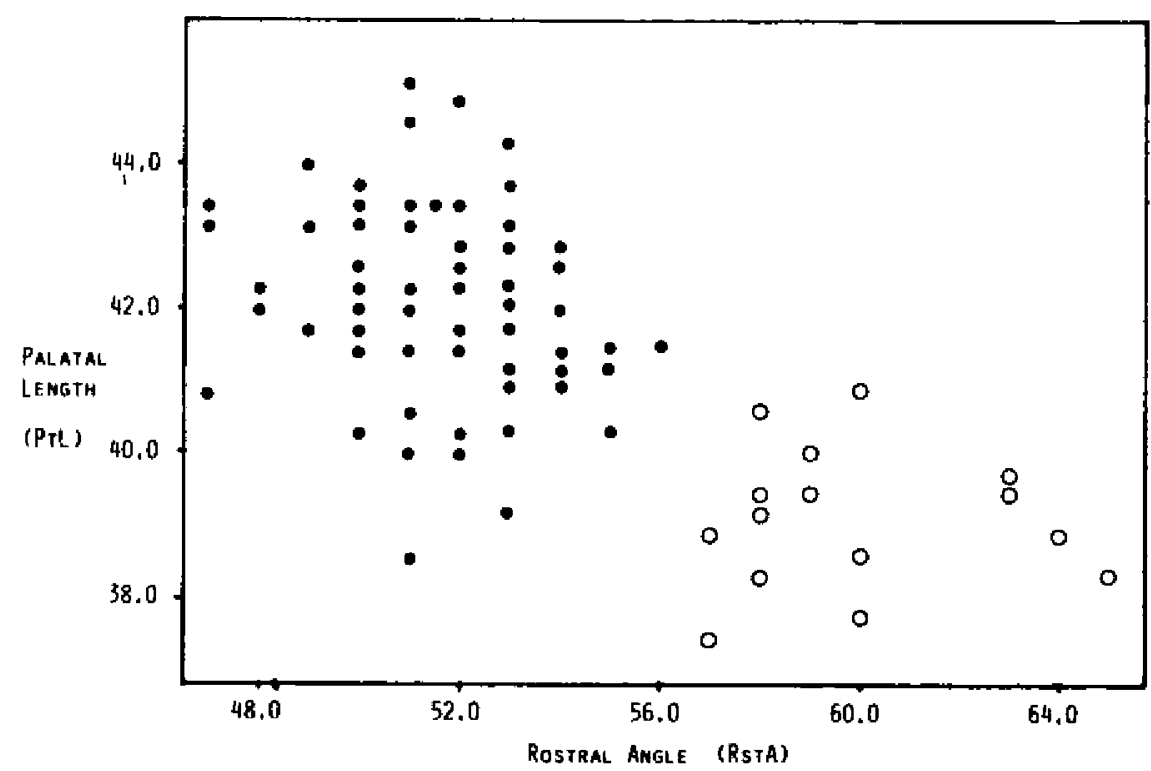

Fig. 7. Plot of palatal length ( $\mathrm{mm}$ ) against rostral angle ( $\left.{ }^{\circ}\right)$ in females of $M$. martes (solid circle) and $M$. foina (open circle) to show the seperation of species. Adults and subadults pooled. 
Table 9

Rotated factor loadings of the first four principal factors (PF) derived from 23 skull measurements of females of $M$. foina from the three age classes pooled. These factors account for $84.1 \%$ of the total variance. VE: percentage of variance explained by each factor. Measurements have been rearranged as in Table 6 .

\begin{tabular}{|c|c|c|c|c|}
\hline Meas. & PF I & PF II & PF III & PF IV \\
\hline $\begin{array}{l}\text { BeC } \\
\text { MnbH } \\
\text { PrL } \\
\text { CbL } \\
\text { BcbH } \\
\text { BcL }\end{array}$ & $\begin{array}{l}0.929 \\
0.757 \\
0.657 \\
0.586 \\
0.568 \\
0.536\end{array}$ & $\begin{array}{r}-0.033 \\
0.207 \\
0.582 \\
0.552 \\
0.180 \\
0.254\end{array}$ & $\begin{array}{r}-0.015 \\
0.136 \\
0.019 \\
0.030 \\
0.473 \\
-0.019\end{array}$ & $\begin{array}{l}0.093 \\
0.064 \\
0.412 \\
0.362 \\
0.418 \\
0.464\end{array}$ \\
\hline $\begin{array}{l}\text { MnbL } \\
\text { MntL } \\
\text { CmL } \\
\text { PtL } \\
\text { MstW }\end{array}$ & $\begin{array}{l}0.505 \\
0.322 \\
0.577 \\
0.348 \\
0.058\end{array}$ & $\begin{array}{l}0.765 \\
0.745 \\
0.623 \\
0.567 \\
0.522\end{array}$ & $\begin{array}{r}0.070 \\
0.010 \\
0.126 \\
0.108 \\
0.240\end{array}$ & $\begin{array}{r}0.374 \\
0.517 \\
0.124 \\
0.477 \\
-0.165\end{array}$ \\
\hline $\begin{array}{l}\text { IorW } \\
\text { BmW } \\
\text { MnbW } \\
\text { EorW } \\
\text { ZyW }\end{array}$ & $\begin{array}{r}0.047 \\
-0.143 \\
0.365 \\
0.067 \\
0.395\end{array}$ & $\begin{array}{r}0.186 \\
0.157 \\
0.201 \\
0.124 \\
0.579\end{array}$ & $\begin{array}{l}0.842 \\
0.796 \\
0.729 \\
0.705 \\
0.633\end{array}$ & $\begin{array}{r}0.185 \\
0.287 \\
0.256 \\
0.061 \\
0.057\end{array}$ \\
\hline $\begin{array}{l}\text { MxtL } \\
\text { RtrW }\end{array}$ & $\begin{array}{r}0.079 \\
-0.034\end{array}$ & $\begin{array}{l}0.259 \\
0.061\end{array}$ & $\begin{array}{r}-0.181 \\
0.413\end{array}$ & $\begin{array}{l}0.782 \\
0.779\end{array}$ \\
\hline $\begin{array}{l}\text { FcL } \\
\text { LcL } \\
\text { PorW } \\
\text { RstA } \\
\text { I.mL }\end{array}$ & $\begin{array}{r}0.306 \\
0.253 \\
0.118 \\
0.068 \\
0.335\end{array}$ & $\begin{array}{r}0.471 \\
0.165 \\
-0.428 \\
-0.259 \\
0.019\end{array}$ & $\begin{array}{l}0.310 \\
0.058 \\
0.084 \\
0.496 \\
0.174\end{array}$ & $\begin{array}{r}0.283 \\
0.499 \\
0.096 \\
0.204 \\
0.438\end{array}$ \\
\hline VE & $23.8 \%$ & $20.7 \%$ & $20.3 \%$ & $19.3 \%$ \\
\hline
\end{tabular}

Table 10

Measurements showing significant differences between adult males of $\boldsymbol{M}$ martes from 4 Polish populations. M: Pomerania and Masurian Lake Region; W: Wielkopolska-Kujawy Lowlands; B: Białowieża Primaeval Forest; $\mathbf{S}$ : Lower and Upper Silesta. Number of skulls in brackets. $F$ values were obtained by ANOVA. Asterisks indicate the degree of significance of pairwise comparison of means by $t$-test between Masurian and Wielkopolska populations (M/W); Masurian and Białowieża (M/B); Wielkopolska and Białowieża (W/B); and Silesia $(\mathrm{B} / \mathrm{S})$

$$
{ }_{* * *} p<0.001 ;^{* *} p<0.01 ;^{*} p<0.05 \text {; - not significant. }
$$

\begin{tabular}{lccccccccc}
\hline Meas. & $F$ & M(16) & W(17) & B(6) & S(11) & M/W & M/B & W/B & B/S \\
\hline PrL & $3.3^{*}$ & 88.5 & 89.5 & 87.7 & 88.3 & $*$ & - & $* *$ & - \\
ZyW & $3.3^{*}$ & 51.5 & 51.8 & 49.6 & 50.8 & - & - & $* *$ & - \\
EorW & $6.4^{* *}$ & 26.1 & 26.3 & 24.0 & 26.0 & - & $* * *$ & $* * *$ & $* *$ \\
IorW & $6.0^{* *}$ & 21.6 & 21.6 & 20.6 & 21.9 & - & $* * *$ & $* *$ & $* * *$ \\
MstW & $8.4^{* *}$ & 40.5 & $\mathbf{4 1 . 2}$ & 39.2 & 40.9 & $*$ & $* * *$ & $* * *$ & $* *$ \\
RtrW & $3.4^{*}$ & 18.0 & 17.7 & 17.3 & 18.0 & - & $* *$ & - & $* *$ \\
BcbH & $5.9^{*}$ & 32.3 & 33.1 & 31.4 & 32.9 & $*$ & - & $* * *$ & $* *$ \\
MndH & $4.3^{*}$ & 24.8 & 25.4 & 24.3 & 25.6 & $*$ & - & $* *$ & $* *$ \\
MndW & $8.9^{* *}$ & 7.2 & 7.6 & 6.4 & 7.3 & $*$ & $* * *$ & $* * *$ & $* * *$ \\
RstA & $5.1^{*}$ & 51.7 & 52.3 & 49.4 & 52.6 & - & $* *$ & $* * *$ & $* * *$ \\
BcC & $3.9^{*}$ & 22.0 & 22.9 & 20.4 & 22.8 & $*$ & - & $*$ & $*$
\end{tabular}


cies. We assumed that RstA is normally distributed and, therefore, the dividing point corresponds to the value of the middle within 2.6 standard deviations (99\% confidence interval) from the mean of RstA in

Table 11

Sample statistics of condylobasal length in adult specimens of $M$. martes in four Polish populations. $\mathrm{n}$ : number of skulls; $\mathrm{X}$ : average; $\mathrm{C}$. V.: coefficient of variation; $\mathrm{O}$. R.:

\begin{tabular}{|c|c|c|c|c|c|c|c|c|}
\hline \multirow{2}{*}{ Population } & \multicolumn{4}{|c|}{ Females } & \multicolumn{4}{|c|}{ Males } \\
\hline & $\mathbf{n}$ & $\overline{\mathbf{x}}$ & C.V. & O.R. & $\mathrm{n}$ & $\overline{\mathbf{x}}$ & C.V. & O.R. \\
\hline $\begin{array}{l}\text { Pomerania and } \\
\text { Masurian Lake Region } \\
\text { Wielkopolska-Kujawy }\end{array}$ & 3 & 78.6 & 1.1 & $77.6-79.4$ & 17 & 85.4 & 1.7 & $82.1-87.7$ \\
\hline $\begin{array}{l}\text { Lowlands } \\
\text { Białowieźa Primaeval Forest } \\
\text { Lower and Upper Silesia }\end{array}$ & $\begin{array}{l}7 \\
2 \\
3\end{array}$ & $\begin{array}{l}79.7 \\
78.4 \\
79.1\end{array}$ & $\begin{array}{l}2.8 \\
1.6 \\
2.3\end{array}$ & $\begin{array}{l}77.5-84.1 \\
77.5-79.3 \\
77.9-81.2\end{array}$ & $\begin{array}{r}18 \\
6 \\
11\end{array}$ & $\begin{array}{l}86.1 \\
84.2 \\
85.0\end{array}$ & $\begin{array}{l}1.5 \\
2.7 \\
1.5\end{array}$ & $\begin{array}{l}83.3-87.8 \\
80.9-87.5 \\
83.7-87.9\end{array}$ \\
\hline
\end{tabular}

Table 12

Differences between $M$. martes in both sexes. $\% \mathrm{D}$ procentage of diference between means in both species $[(\overline{\mathbf{x}} M$. foina $-\overline{\mathbf{x}} M$. martes $) / M$. martes $\times 100]$ $\overline{\mathrm{X}}_{\mathrm{w}_{\mathrm{D}}}$ : average of $\% \mathrm{D}$ in the 23 measurements. $F$ values were obtained by ANOVA between the two species, adults and subadults pooled. $F$ values were highly signifcant $(p<0.001$, unless otherwise marked. ${ }^{*} p<0.01 ;{ }^{*} p<0.05-$ not significant.

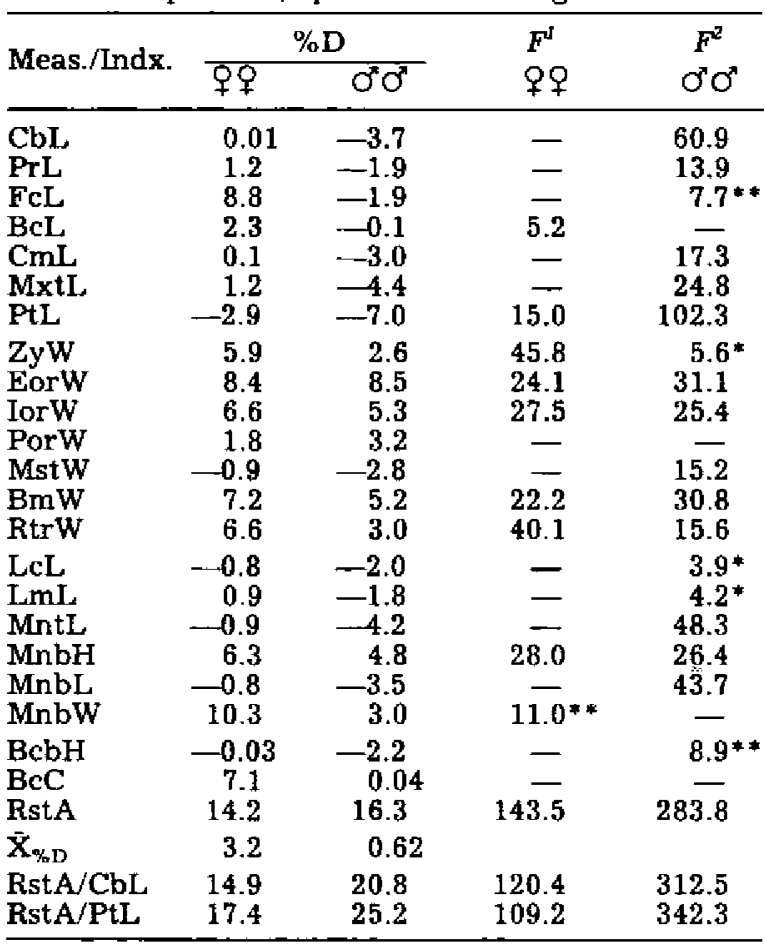

${ }^{1} \mathrm{df}=1,69 ;{ }^{2} \mathrm{df}=1,119$; 


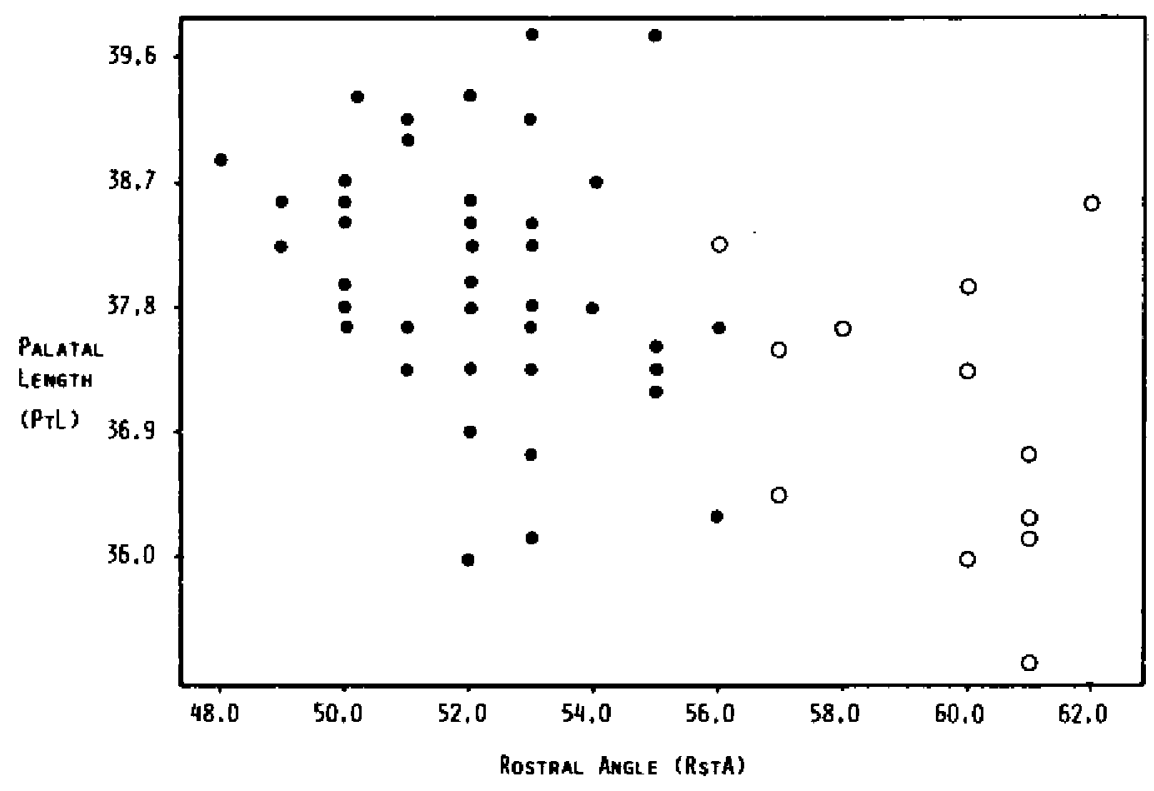

Fig. 8. Plot of palatal length $(\mathrm{mm})$ against rostral angle $\left({ }^{\circ}\right)$ in males of $M$. martes (solid circle) and $M$. foina (open circle) to show the seperation of species. Adults and subadults pooled.

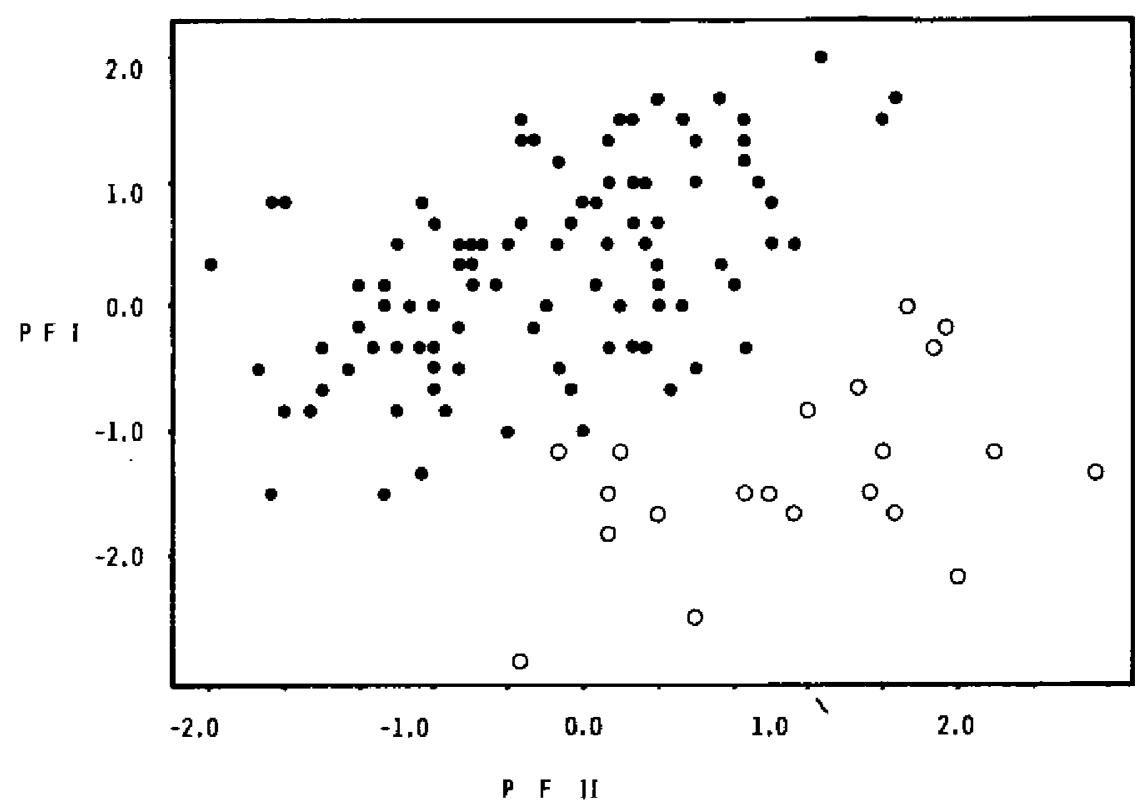

Fig. 9. Plot of Principal Factor I (PF 1), (related to skull length) against Principial Factor II (PF), (related to skull width) in females of $M$. martes (solid circle) and $M$. foina (open circle) to show the seperation of species. Adults and subadults pooled. 
each species (all groups of age and sex pooled). This results in a value of $54.9^{\circ}$, above which, specimes should be classified as $M$. foina and vice versa. Using this criterion, $98 \%$ of the skulls were correctly classified, $97 \%$ of $M$. martes and $100 \%$ of $M$. foina (Fig. 6 ).

Principal factor analysis yielded three PF which explained 87.9 and $85.5 \%$ of the total variance in males and females, respectively (Table 13). Results were similar in both sexes, PF I was related to skull and mandible length, while PF II joined width measurements, RstA, MndH and MndW. PF III showed high loadings from traits related to the neurocranium, such as $\mathrm{BcC}, \mathrm{BcbH}$, and PorW, which are characterized by little specific differences, and consequently with also little amount of variance explained. BcI, $\mathrm{L} m \mathrm{~mL}, \mathrm{MstW}$ and $\mathrm{LcL}$ are not clearly contributing to any of the three factors (Table 13). Attention must be paid to the fact that in the three PF, negative and positive loadings occur simultaneously. This suggests that, rather than size features, a general shape factor synthesized the differences between species. To estimate the effectiveness of PF to separate both species, PF I was plotted against PF II (Figs 9, 10).

Table 13

Rotated factor loadings of the first three principial factors (PF) showing differences between $M$. martes and $M$. foina in both sexes, adults and subaciult specimens pooled. These factors account for $85.5 \%$ and $87.9 \%$ of the total variance in females and males, respectively. Measurements have been rearranged as in Table 6

\begin{tabular}{|c|c|c|c|c|c|c|}
\hline \multirow{2}{*}{ Meas. } & \multicolumn{3}{|c|}{ Females } & \multicolumn{3}{|c|}{ Males } \\
\hline & PF I & PF Ш & PF III & PF I & PF II & PF UII \\
\hline $\begin{array}{l}\text { CbL } \\
\text { MntL } \\
\text { PrL } \\
\text { MndL. } \\
\text { CmL } \\
\text { PtI } \\
\text { FcL } \\
\text { BmW } \\
\text { BcL }\end{array}$ & $\begin{array}{l}0.842 \\
0.797 \\
0.795 \\
0.777 \\
0.771 \\
0.762 \\
0.587 \\
0.562 \\
0.558\end{array}$ & $\begin{array}{r}0.194 \\
-0.014 \\
0.340 \\
-0.032 \\
0.130 \\
0.372 \\
0.249 \\
0.123 \\
0.345\end{array}$ & $\begin{array}{r}0.059 \\
-0.077 \\
0.069 \\
-0.006 \\
0.105 \\
0.102 \\
0.037 \\
0.090 \\
-0.031\end{array}$ & $\begin{array}{l}0.951 \\
0.892 \\
0.877 \\
0.943 \\
0.711 \\
0.855 \\
0.683 \\
0.634 \\
0.449\end{array}$ & $\begin{array}{r}-0.024 \\
-0.004 \\
0.293 \\
0.136 \\
0.022 \\
-0.225 \\
0.081 \\
-0.065 \\
0.421\end{array}$ & $\begin{array}{r}0.071 \\
-0.064 \\
0.152 \\
-0.025 \\
0.048 \\
0.057 \\
0.180 \\
0.028 \\
0.264\end{array}$ \\
\hline $\begin{array}{l}\text { ZyW } \\
\text { RstA } \\
\text { EorW } \\
\text { IorW } \\
\text { RtrW } \\
\text { CmW } \\
\text { MndW } \\
\text { MndH }\end{array}$ & $\begin{array}{r}0.267 \\
-0.289 \\
-0.032 \\
0.091 \\
0.385 \\
0.210 \\
0.541 \\
0.345\end{array}$ & $\begin{array}{l}0.877 \\
0.848 \\
0.770 \\
0.751 \\
0.683 \\
0.681 \\
0.654 \\
0.646\end{array}$ & $\begin{array}{l}0.068 \\
0.143 \\
0.117 \\
0.135 \\
0.080 \\
0.042 \\
0.010 \\
0.320\end{array}$ & $\begin{array}{r}0.185 \\
-0.599 \\
-0.055 \\
-0.031 \\
0.182 \\
-0.147 \\
0.438 \\
0.130\end{array}$ & $\begin{array}{l}0.798 \\
0.671 \\
0.856 \\
0.845 \\
0.734 \\
0.498 \\
0.733 \\
0.645\end{array}$ & $\begin{array}{l}-0.040 \\
0.161 \\
0.064 \\
0.025 \\
0.086 \\
0.227 \\
0.049 \\
0.334\end{array}$ \\
\hline $\begin{array}{l}\text { BebH } \\
\text { BcC } \\
\text { PorW }\end{array}$ & $\begin{array}{r}0.303 \\
0.037 \\
-0.173\end{array}$ & $\begin{array}{l}0.029 \\
0.191 \\
0.132\end{array}$ & $\begin{array}{l}0.828 \\
0.776 \\
0.726\end{array}$ & $\begin{array}{r}0.547 \\
0.193 \\
-0.032\end{array}$ & $\begin{array}{r}0.209 \\
-0.003 \\
0.098\end{array}$ & $\begin{array}{l}0.535 \\
0.944 \\
0.602\end{array}$ \\
\hline $\begin{array}{l}\text { LmL } \\
\text { MstW } \\
\text { LeL }\end{array}$ & $\begin{array}{l}0.406 \\
0.389 \\
0.313\end{array}$ & $\begin{array}{r}0.110 \\
0.169 \\
-0.045\end{array}$ & $\begin{array}{r}0.181 \\
-0.093 \\
0.303\end{array}$ & $\begin{array}{l}0.449 \\
0.659 \\
0.447\end{array}$ & $\begin{array}{l}0.049 \\
0.300 \\
0.175\end{array}$ & $\begin{array}{l}0.104 \\
0.156 \\
0.154\end{array}$ \\
\hline VE & $37.0 \%$ & $31.0 \%$ & $17.5 \%$ & $45.5 \%$ & $29.4 \%$ & $12.9 \%$ \\
\hline
\end{tabular}


Stepwise discriminant analysis was performed, using a data matrix which included all groups of skulls. After five steps, the DF extracted explained $100 \%$ of the total variance, classifying correctly all cases (Fig. 11). Out of the five characters selected for the DF, three were related to width of the skull (MstW, RstA and Rtrw) and the other two

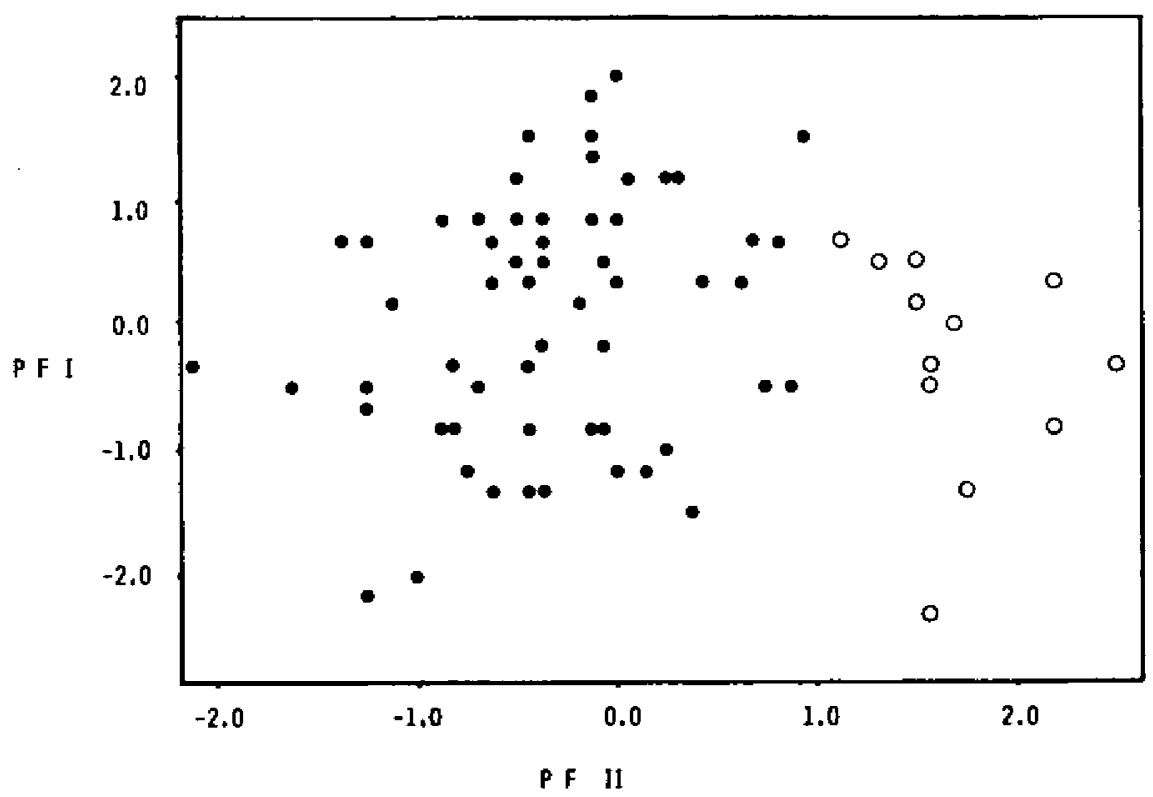

Fig. 10. Plot of Principal Factor I (PF I), (related to skull length) against Principial Factor $\Pi(\mathrm{PF})$, (related to skull width) in males of $M$. martes (solid circle) and $M$. foina (open circle) to show the seperation of species. Adults and subadults pooled.

$\mathrm{C}=16.35+0.96(\mathrm{MstH})+0.86(\mathrm{MHDH})+0.84(\mathrm{RsTA})-0.70(\mathrm{RsTW})-0.60(\mathrm{BcBH})$

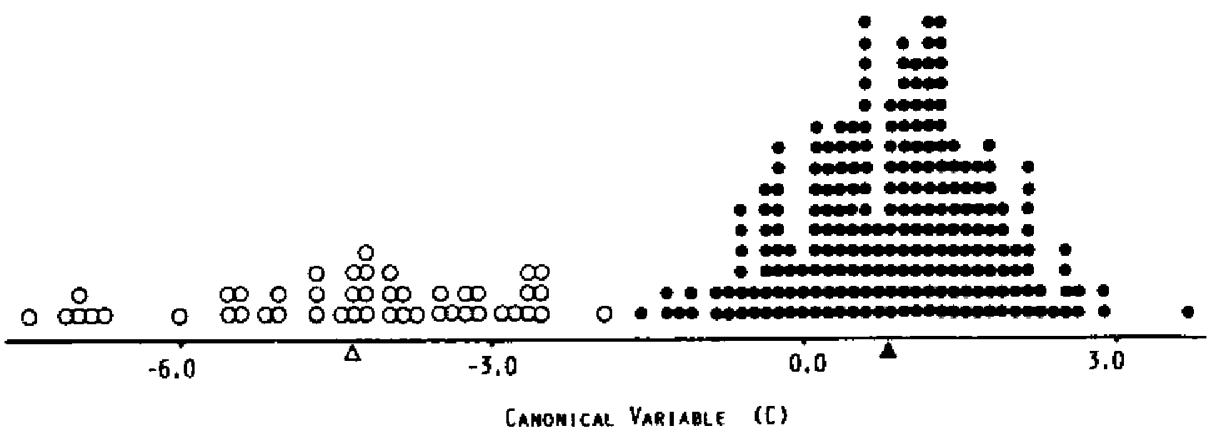

Fig. 11. Histogram of canonical variable scores of the whole collection o skulls to show the seperation of species by stepwise discriminant analysis. On the top the histogram, coefficient of measurements included in the cancional variable. Triangles indicate group centroids. Solid circle: $M$. martes; open circle; $M$. foina. 
to height of the skull ( $\mathrm{MndH}$ and $\mathrm{BcbH}$ ). The highest contribution to the discriminant function was not from RstA but from MstW, suggesting that these two measurements are correlated, and therefore, some of the species discriminating usefulness of RstA is explained by its correlation with MstW.

\section{DISCUSSION}

\subsection{Methods}

Integrated use of univariate and multivariate methods proved to be essential for the study of variability (Gould \& Johnston, 1972; Pankakoski \& Nurmi, 1986). Univariate analysis is needed because it provides specific information about the variation of particular traits or regions of the skull which may have special interest for taxonomical or biological reasons. Moreover, comparison of the results with other species or other studies is only possible through single characters. On the other hand, multivariate methods enabled a better understanding of biological processes, which are more likely to be multidimensional complexes (Gould \& Johnston, 1972; Wiig, 1985).

To describe the relationships between characters, factor analysis (Atchley, 1971 a; Wiig, 1985) was preferred instead of correlation of measurements by minimum squares (Cabon-Raczyńska, 1964; Buchalczyk \& Ruprecht, 1977; Pankakoski \& Nurmi, 1986). The main advantages of this method in comparison to simple correlation were: (1) different sample size of the groups compared (age, sex, populations, species) does not alter the results and meaning of the factors constructed, (2) the amount of variance explained by each association of measurements (factor) can be calculated, (3) the associations between measurements does not have a fixed structure but varies according to the different sources of variation analyzed (Tables 6, 7, 13), (4) instead of the pairwise associations between measurements provided by the coefficient of correlation, PFA present multiple set of measurements in meaningful subsets.

\subsection{Sex Dimorphism}

Skull proportions showed little sex dimorphism in both species. Differences between males and females were mainly related to general size of the skull, as occurs in most carnivores (Ewer, 1973). The average difference between sexes in the 23 characters indicate that sex dimorphism was more apparent in $M$. martes than in $M$. foina (Table 3 ). However, in both species size differences between sexes were low in comparison with other mustelids. Using condylobasal length as a meas- 
ure of skull size (Hagmeier, 1958; Ralls \& Harvey, 1985), adult females are $18.2 \%$ smaller in Mustela putorius (Buchalczyk \& Ruprecht, 1977), $16.3 \%$ in Martes pennanti (Erxleben, 1773) (Anderson, 1970), 15.5\% in Mustela erminea Linnaeus, 1758 (Hall, 1951), while in $M$. martes and $M$. foina females are, respectively, only $8.7 \%$ and $4.8 \%$, smaller than males (Table 3). Even a closely related species like Martes zibellina (Linnaeus, 1758), has larger sex dimorphism (10.0\%, after Anderson 1970).

Two principal hypothesis are maintained to explain sexual dimorphism in mustelids. One postulates that size differences between sexes reduce intraspecific competition for food (Brown \& Lasiewski, 1972; Powell, 1979; Moors, 1980; Powell \& Leonard, 1983), while the other hypothesis claims that fighting between males for coupling in polygamous species had selected for increased male size (Erlinge, 1979; Moors, 1980). Summarizing the first hypothesis as a direct correlation between food competition and sex dimorphism, the results of this study would fully support this argument, knowing the generalistic feeding strategy of both species (Erlinge, 1986), specially $M$. foina. Moreover, detailed studies on the food of $M$. martes did not show differences between the food habits of males and females (Pulliainen, $1981 \mathrm{~b} ;$ Marchesi, 1989).

Masticatory apparatus, in particular weight and height of the mandible, exhibited the most significant sex dimorphism in both species of martens (Table 3), as found by Rossolimo \& Pavlinov (1974) in M. martes. This is a common feature small mustelids (Yurgenson, 1947; Petrov, 1956), and has been explained also as a consequence of their sexual differences in food habits. Nevertheless, Wiig (1989) explaining similar features of the sexual dimorphism in Gulo gulo (Linnaeus, 1758), suggested that female's differences in the masticatory apparatus might be just a morphological expression of maintaining the same chewing efficiency in a smaller skull, since food habits are the same in individuals of both sexes. Such an explanation seems to be also valid for the two species of martens studied.

\subsection{Age Variation}

Proportions between measurements exhibited important age changes, suggesting allometric growth of the skull. Clear age changes were observed in indices which combine PorW and $\mathrm{BcbH}$, showing negative rate, with other characters. Weight of the mandible and zygomatic width of the skull were the traits exhibiting most significant age variation (Tables 4,5 ) as in most of mammals (Yablokov, 1974).

The division of the material into three age calsses was appropriate, 
regarding the differences found between groups. Further division of the material into more age groups was not only limited by the number of skulls avilable, but also by the difficulties of determining new groups using only external features. Methods commonly used in carnivores such as suture obliteration are not suitable for martens since most sutures are completely closed at very early age (Ryabov, 1962). On the other hand, Brown (1983) analyzing the age variation in $M$, americana (Turton, 1806) and having absolute age determined by cementum incremental lines, suggested also the division of the material into three age groups for studies of cranial variation in martens.

The growing process of the marten skull is highly precocious. Ryabov (1962) found that $\mathrm{CbL}$ of males of $M$. martes from Caucasus region attain $88.6 \%$ of adult size $(86.6 \%$ in the case of $\mathrm{ZyW})$ at only two months of age. Two important consequences for our study are derived from this finding. The first one is that skulls older than five months (the material used in this study) reflect only a small fragment of the whole postnatal growth of martens. Secondly, age groups used correspond to a period of stabilized growth process, when the animals are said to be already fully grown.

For these reasons, differences between juvenile and subadults exhibited similar degree of significance than differences between subadults and adults, especially on males (Tables 4,5 ). This growing of the skull after the first year of life agrees also with Rossolimo \& Pavlinov (1974), who observed a slow. growth process prolonged throughout the life of the individual in $M$. martes, and also the existence of significant sex differences in the growth rates, females growing more rapidly than males. Nevertheless, our results for particular traits differ from that study because authors mentioned above used $\% \mathrm{D}$ between means from both sexes as the main indicator of the significance of age variation, instead of considering $F$ values obtained by ANOVA. Also in other carnivores like the European lynx (Wiig \& Andersen, 1986) the skull of adult individuals continues to grow, specially in males.

In both species more than $60 \%$ of the total age variation was explained only by two principal factors, one joining most length traits, the other joining width traits. Noticeable differences between species were found in the relative importance of each of these two factors for the skull growth. In $M$. martes length factor was more important, explaining $51.3 \%$ of the total variance, while the width factor only accounted for $18.9 \%$ (Table 6 ). On the contrary, in $M$. foina length and width factors showed similar amount of varinace explained $(29.9 \%$ and $31.7 \%$, respectively, Table 8 ), the width factor being a little more important. 
Postorbital width was characterized by a progressive narrowing with age in both species. Previous studies in $M$. martes (Mal'dzhyunaite, 1957), and $M$. foina (Röttcher, 1965) agree with this results, although the significance of the age variability of this trait in $M$. martes and $M$. foina was first quantified in this study (Tables 4,5 ). Negative growth of postorbital width is also a common feature in other mustelids, like Martes americana (Brown, 1983), $M$. putorius (Buchalczyk \& Ruprecht, 1977), and also in other mamals e.g. Ondatra zibethicus (Linnaeus, 1766) (Pankakoski \& Nurmi, 1986). Age variation of this measurement must be related to overall changes in the brain case capacity (Wiig, $1982)$, since the results of factor analysis indicated that this measurement is associated with $\mathrm{BcC}$ and $\mathrm{BcbH}$.

\subsection{Variability between Populations in $M$. martes}

The observed differences between the Białowieża population and the rest of Polish territory, accords with the north-south axis of skull increase found by Reig (1989). However, the similarity of Wielkopolska and Masurian samples (Table 10 ) suggest that results are mainly due to distinctive features of the Bialowieża population. Similar results were also observed in Mustela putorius (Buchalczyk \& Ruprecht, 1977), and in two species of Erinaceus (Ruprecht, 1972). Among the eleven traits showing significant differences between populations, MndW, ZyW, and EorW are charactreized also by an important age variation, while others like RstA or $\mathrm{BcC}$ showed low age variation. This may suggest that the differences observed between populations are not only related to environmental factors affecting skull growth, but with phenetical peculiarities of the Białowieża population.

\subsection{Species Differentiation}

Rostral angle (RstA), despite being a trait very seldom used in craniometrical studies of mammals, proved to have an important taxonomic value in European martens since it allowed the distinction between $M$. martes and $M$. foina. Fully accurate identification of both species is, however, better achieved using non-metrical traits (Anderson, 1970; Wolsan et al., 1985; Steiner \& Steiner, 1986). Among most frequently used metrical characters for this purpose are the distance between foramina mentalia (Gaffrey, 1953) which is not fully precise because of the great variability of these openings and the index between some interorbital measurements (Altuna, 1973) which has the main disadvantage of being based on a graphic representation. However, using RstA, no overlapping between species was obtained when groups 
of the same sex and age were matched (Tables 1,2). The accuracy of this trait was high; $98 \%$ of the complete collection of skulls ( $n=283$ ) were correctly calassified into species using a dividing point of $54.9^{\circ}$ (Fig. 6). Moreover, RstA has the advantage of exhibiting very little variation with age or sex, not significant in most of the cases.

Considering the association of measurements by principal factor analysis, RstA seems to be related to width of the skull (Tables 6, 7, 8, 9 ), although it is not coincident with width measurements as concerns pattern of age and sex variation (Tables $3,4,5$ ). An attempt was made to separate both species using indexes based on combinations of another measurements than RstA, but related to this region of the skull, as ZyW, RtrW, FcL and PtL (Fig. 3). However, results were not satisfactory, suggesting that RstA reflects a more camplex ratio between width and length of the skull.

The final conclusion in that the patterns of variability are distinct in two species showing remarkable morphological similarities. Differences were concerning not only particular traits reflecting the most significant changes related to sex or age, but also the main pattern of skull variability. Most of the morphological changes in the skull of martens due to sex or age variation were explained by the association of measurements into a length and a width factor. However, in each species the relative importance of these factors to explain the variability observed was different, and in some cases opposite. The most substantial testimony of this argument was the clear separation of species by plotting these two factors (length and width of the skull) against each other (Figs. 9, 10).

An alternative explanation for distinct variability patterns in these marten species, besides their different ecological characteristics, is connected with the phylogeny of the genus Martes, since both species are considered to belong to separate phylogenetic groups. $M$. martes is much closer to M. zibellina, M. melampus (Wagner, 1841), and M. americana than to $M$. foina (Anderson, 1970; Wolsan, 1986).

Acknowledgments: We are indebted to M. Sci. Tadeusz Buchalczyk, who generously provided a considerable amount of data on skull measurements. We thank also Prof Zdzislaw Pucek for his continuous support and qualified comments. Dr Mieczysław Wolsan provided useful help for the study of the skulls from the Institute of Experimental and Systematic Zoology (Cracow). We are also very grateful to an anonymous reviewer who gave valuable suggestions to the first version of the manuscript. Sojourn in Białowieża of S.R. was supported by a UNESCO/PAS fellowship 1985-1986, and by CSIC/PAS exchange program in 1989 , whereas Iberia Airlines kindly covered travel expenses.

\section{REFERENCES}

1. Altuna J., 1973: Distinción craneal entre la Marta (Martes martes) y la Foina (Martes foina) (Mammalia). Munibe, 25: 33-38. 
2. Anderson E., 1970: Quaternary evolution of the genus Martes (Carnivora, Mustelidae). Acta Zool. Fenn., 130: 1-132.

3. Atchley W., 1971a: A statistical analysis of geographic variation in the pupae of three species of Culicoides (Diptera: Ceratopogonidae). Evolution, 25: 51-74.

4. Atchley W., 1971 b: A comparative study of the cause and significance of morphological variaton in adults and pupae of Culicoides: A factor analysis and multiple regression study. Evolution, 25: $563-583$.

5. Baker A. J., Peterson R. L., Eger J. L. \& Manning T. H., 1978: Statistical analysis of geographic variation in the skull of the arctic hare (Lepus arcticus). Can. J. Zool., 56: $2067-2082$.

6. Bree P. J. H. van, Mensh P. J. A. van \& Utrecht W. L. van, 1970: Sur le dimorphisme sexuel des canines chez la Foine, Martes foina (Erxleben, 1777). Mammalia, 34: 676-682.

7. Brown M., 1983: A morphometric analysis of sexual and age variation in the American marten, Martes americana (Camivora: Mustelidae). $\mathrm{Ph} \mathrm{D}$. Thesis, University of Toronto, Canada, 1-190.

8. Brown J. H. \& Lasiewski R. C., 1972: Metabolism of weasels: the cost of being long and thin. Ecology 53: 939-943.

9. Buchalczyk T. \& Ruprecht A. L., 1977: Skull variability of Mustela putorius Linnaeus, 1758. Acta theriol.,22: 87-120.

10. Caboń-Raczyńska K., 1964: Correlations of skull measurements of Lepus europaeus Pallas, 1778. Acta theriol., 8: 207-216.

11. Dixon M., 1987: BMDP Statistical Software, 1985. Berkeley: University of California Press. 1-940.

12. Erlinge S., 1979: Adaptative significance of sexual dimorphism in weasels. Oikos, 28: $32-42$.

13. Erlinge S., 1986: Specialists and generalists among the mustelids. Lutra, 29: 5-11.

14. Ewer R. F., 1973: The carnivores. London. Weidenfeld and Nicholson, 1- 494.

15. Gaffrey, G., 1953: Die Schădel der mitteleuropäischen Sāugetiere. Abh. Ber. Staatl. Mus. Tierk.-Forschungsinstitut-Dresden, 21: 5-123.

16. Gerasimov S., 1985: Species and sex determination of Martes martes and Martes foina by use of systems of craniometrical indices developed by stepwise discriminant analysis. Mammalia, 49: 235-248.

17. Gould S. J. \& Johnston R. F., 1972: Geographic variaton. Annual Rev. Ecol. Syst., 3: $457-498$.

18. Habermehl K. H. \& Röttcher D., 1967: Die Möglichkeiten der Altersbestimmung beim Marder und Lltis. Ztschr. Jagdwiss., 13: 89-102.

19. Hagmeier E. D., 1958: Inapplicability of the subspiecies concept to North American martens. Syst. Zool, $7: 1-7$.

20. Hall E. R., 1951: American weasels. University of Kansas Publication No. 4: $1-466$.

21. Heptner V. G. \& Morozova-Turova L. G., 1951: Materialy k izuceniju volka s opisanem novogo metoda ustanovlenija vozrastnoj izmencivosti mlekopitajuščih. Sb. tr. Gos. Zool. Muzeja MGU Moskva, 7: 5-14.

22. Heptner V. G., Naumov N. P., Jurgeson P. B., Sludskij A. A., Cirikova A. F. \& Bannikov A. G., 1967: Mlekopitajuščie Sovietskogo Sojuza. Niorskie korovy i hiščnye. Izd. Vysšaja \$kola, (ed.) V. G. Heptner \& N. P. Naumov, 2: 1-1004. Moskva.

23. Mal'dzhyunaite S. A., 1957: Age determination and age structure of pine martens in Lithuania. [In: "Biology of the Mustelids: some Soviet Research". Vol. 1, Ed. C. M. King 1975]: 132-144.

24. Marchesi P., 1989: Ecologie et comportement de la martre (Martes martes L.) dans le Jura Suisse. These de doctorat Univ. Neuchàtel. Switzerland. 1-185. 
25. Moors P. J., 1980: Sexual dimorphism in the body size of mustelids (Mammalia: Carnivora): the role of food habits and breeding systems. Oikos, 34: 147-158.

26. Pankakoski E. \& Nurmi K., 1986: Skull morphology of Finnish muskrats: geographic variation, age differences and sexual dimorphism. Ann. Zool. Fennici, 23: 1-32.

27. Pavlinov I., 1977: Age related changes in the skull characters of Martes martes L. (Mammalia: Mustelidae) in the postnatal period. Byull. Mosk. O-va Isp. Prir. Ot. Biol., 82, 5: 33-49. [In Russian with English summary]

28. Petrov O. V., 1956: Sexual dimorphism in the skull of Mustela erminea L. [In: "Biology of the Mustelids: some Soviet Research". Vol. 1, Ed. C. M. King 1975], 55-78.

29. Powell R. A., 1979: Mustelid spacing patterns: variations on a theme by Mustela. Ztschr. Tierps., 50: 153-165.

30. Powel R. A. \& Leonard R. D., 1983: Sexual dimorphism and energy expenditure for reproduction in female fisher Martes pennanti. Okios, 40:166-174.

31. Pulliainen E., 1981a: Winter habitat selection, home range and movements of the pine marten (Martes martes) in a Finish Lapland forest. [In: Worldwide Furbearer Conf. Proc. Vol. 2, Chapman J. A., Pursley D. Ed.]: 1068 -1087.

32. Pulliainen E., 1981b: Food and feeding habits of the pine marten in Finnish Forest Lapland in winter. [In: Worldwide Furbearer Conf. Proc. Vol. 1, Chapman J. A., Pursley D. Ed.]: $580--598$.

33. Ralls K. \& Harvey P. H., 1985: Geographic variation in size and sexual dimorphism in North American weasels. Biol. J. Linn. Soc., 25: 119-167.

34. Reig S., 1989: Morphological variability of Martes martes and Martes foina in Europe. Ph D. Thesis, Jagielionian University. Cracow, Poland. 1-145.

35. Rossolimo O. \& Pavlinov L., 1974: Sexual dimorphism in the development, size and proportions of the skull in the pine marten (Martes martes Linn.: Mammalia, Mustelidae). [n: "Biology of the Mustelids: some Soviet Research". Vol. 1, Ed. C. M. King 1975]: 180-191.

36. Röttcher D., 1965: Beiträge zur Altersbestimmung bei Nerz, Steinmarder und Iltis. Inaugural-Dissertation zur Erlangung des Doktorgrades bei der Veterinärmedizinischen Fakultät der Justus Liebig-Universität zu Giessen: 1-83.

37. Ruprecht A. L., 1972: Correlation structure of skull dimensions in European hedgehogs. Acta theriol., 17: 419--442.

38. Ryabov L. S., 1962: The morphological development of Caucasian pine martens and stone martens in relation to age determination. [In: "Biology of the Mustelids: some Soviet Research". Vol. 1, Ed. C. M. King 1975]: 145-157.

39. Sokal R. R. \& Rinkel R. C., 1963: Geographic variation of alate Pemphigus populi-transversus in Eastern North America. Univ. Kans. Sci. Bull, 42: 1067-1097.

40. Steiner H. M. \& Steiner F. M., 1986: Die nicht-metrische Unterscheidung von Schädeln mitteleuropäischer Baum- und Steinmarder (Martes martes und Martes foina, Mammalia). Ann. Naturhist. Mus. Wien, 88/89 B: 267-280.

41. Vericad J. R., 1970: Estudio faunistico y biologico de los mamiferos del Pirineo. Publ. Centro Pirenaico Biol. Exper., 4: 7-232.

42. Wig $\emptyset$. \& Andersen T., 1986: Sexual size dimorphism in the skull of Norwegian Iynx. Acta theriol., 31: 147-155

43. Wiig $\emptyset$., 1982: Bone resorption in the skull of Mustela vison. Acta theriol., 27: $358-360$.

44. Wiig $\emptyset$., 1985: Multivariate variation in the feral American mink (Mustela vison) from Southern Norway. J. Zool., Lond. (A), 206: 441-452.

45. Wiig Ø., 1989: Craniometric variation in Norwegian wolverines Gulo gulo L. Zool. J. Linn. Soc., 95: 177-204. 
46. Wolsan M., Ruprecht A. L. \& Buchalczyk T., 1985: Variation and asymmetry in the dentition of the pine and stone martens (Martes martes and $M$. foina) from Poland. Acta theriol., 30: 79-114.

47. Wolsan M., 1986: Morphological variation of the first upper molar in the genus Martes (Carnivora, Mustelidae). Mem. Mus. natn. Hist. nat. Paris, (serie C), 53: 241-254, 1988 .

48. Yablokov A. V., 1974: Variability of mammals. Amerind Publ. Co., New Delhi, 1350.

49. Yurgenson P. B., 1947: Sexual dimorphism in feeding as an ecological adaptation of a species. [In: "Biology of the Mustelids: some Soviet Research". Vol. 1, Ed. C. M. King 1975]: $79-83$.

Received 24 June 1988, Accepted 1 August 1989.

Santiago REIG i Andrzej L. RUPRECHT

ZMIENNOSC MORFOLOGICZNA CZASZKI KUNY LESNEJ I KAMIONKI Z POLSKI

Streszczenie

Zbadano zmiennosce 23 pomiarów czaszkowych oraz kilku wskaźników ilorazowych na materiale 236 czaszek $M$. martes (133 samców i 103 samic) i 47 czaszek $M$. foino ( 25 samcow i 22 samic) z terenu Polski w aspekcie plci i wieku. Liczniejszy material $M$. martes badano w 4 grupach regionalnych (Pojezierze Pomorskie i Mazurskie, Nizina Wielkopolsko-Kujawska, Puszcza Białowieska oraz Górny i Dolny Śląsk). Wyróżniono 3 grupy wiekowe $w$ oparciu o datę odlowu i stan zachowania szwów czaszkowych a także stopień starcia zębów i wyksztalcenia grzebienia strzałkowego. Oba gatunki kun odznaczają sie wyraźnym dymorfizmem plciowym w wymiarach czaszki - samce są większe od samic przeciętnie o $12,1 \%$ u kuny leśniej i 9,5\% u kamionki (Tabela 3). Zaznacza się on przede wszystkim w okolicach czaszki związanych funkcjonalnie z przyjmowaniem pokarmu. Najsilniej zaznaczone różnice wiekowe obserwowano w przypadku masy i długości żuchwy (MnbW i MnbL) oraz szerokosci jarzmowej (ZyW), wyraźniej widoczne u kuny lesnej (Tabela 4 i 5 ). Zmiany wiekowe czaszki badane metoda analizy czynnikowej u $M$. martes widoczne sa $z$ reguly $w$ pomiarach długosciowych, w mniejszym stopniu $w$ szerokościowych (lącznie oba czynniki stanowia $70 \%$ ). U $M$. foina natomiast metoda analizy czynnikowej ujawniła tendencje odwrotna, $z$ tą różnica że oba czynniki pozostają w proporcji 1:1 (Tabela 6-8). Obydwa gatunki kun najlepiej różnicuje kąt rostralny (RstA), który u $M$. martes jest ostry u $M$. foina bardziej rozwarty. Porownanie w obrębie trzeciej grupy wiekowej samców ujawnilo 11 różnic populacyjnych w pomiarach czaszki między kunami lesnymi z Puszczy Bialowieskiej i pozostałymi trzema populacjami (Pojezierze Pomorskie i Mazurskie, Nizina Wielkopolsko-Kujawska oraz Dolny i Gorny Sląsk) (Tabela 10). 